\title{
Optical Gain and Co-Stimulated Emissions of Photons and Phonons in Indirect Bandgap Semiconductors
}

\author{
M. J. CHEN, C. S. TSAI ${ }^{1,2}$ and M. K. WU \\ Department of Materials Science and Engineering, National Taiwan University, Taipei 106, Taiwan \\ ${ }^{1}$ Department of Electrical Engineering and Computer Science and Institute of Surface and Interface Science, \\ University of California, Irvine, CA 92687, U.S.A. \\ ${ }^{2}$ Institute of Electrooptics Engineering, National Taiwan University, Taipei 106, Taiwan
}

(Received February 24, 2006; accepted June 5, 2006; published online August 22, 2006)

A model calculation on optical gain and co-stimulated emission of photons and phonons in indirect bandgap semiconductors such as silicon is presented. An analytical expression for optical gain via phonon-assisted optical transitions in indirect bandgap semiconductors is presented. Population inversion can occur when the difference between the quasi-Fermi levels for electrons and holes is greater than the photon energy. The rate equations and their steady state solutions for electron, photon, and phonon involved in the phonon-assisted optical transitions are presented. It is shown that co-stimulated emissions of photons and phonons will occur when the threshold condition for laser oscillation is satisfied. The magnitude of optical gain in bulk crystalline silicon is calculated and shown to be smaller than the free carrier absorption at room temperature. However, it is shown, for the first time, that the optical gain is greater than the free carrier absorption in bulk crystalline silicon at the temperature below $23 \mathrm{~K}$. Thus, the calculation predicts that the co-stimulated emissions of photons and phonons could take place in bulk crystalline silicon at the low temperature. [DOI: 10.1143/JJAP.45.6576]

KEYWORDS: indirect bandgap semiconductors, crystalline silicon, optical gain, co-stimulated emission of photons and phonons, phonon-assisted optical transition, laser oscillation, nanostructured PN junction diode

\section{Introduction}

Silicon is the dominant semiconductor material in ultralarge-scale-integration (ULSI) electronics, and it is also the desirable substrate material for realization of integrated optoelectronics. ${ }^{1,2)}$ However, until the most recent realization of Raman-effect based laser, ${ }^{2,3)}$ it was generally believed that silicon's indirect bandgap would prevent its use as efficient light emitters and lasing media. Numerous earlier approaches, ${ }^{5-7)}$ such as silicon nanocrystals, ${ }^{8,9)} \mathrm{Si}$ / $\mathrm{SiO}_{2}$ supperlattices, ${ }^{10)}$ porous silicon, ${ }^{12)}$ erbium-doped silicon, ${ }^{13,14)}$ and periodic nanopatterned crystalline silicon ${ }^{15}$ with emphases on photoluminescence aspects, have been attempted with some progresses toward overcoming this difficulty. For the approaches with emphases on electroluminescence aspects, high efficiency electroluminescence from silicon light-emitting diodes (LED), in which the emission wavelength corresponds to the silicon bandgap energy, was reported. ${ }^{16-18)}$ Room-temperature electroluminescence at bandgap energy was also observed in metaloxide-semiconductor (MOS) tunneling diodes on silicon. ${ }^{19)}$ Recently, stimulated emission at the wavelength corresponding to the silicon bandgap energy was observed in a silicon nanostructured pn junction diode using current injection at room temperature. ${ }^{20)}$ Most recently, field-effect electroluminescence from silicon nanocrystals in a floating-gate transistor structure was reported. ${ }^{21}$ On the theoretical study of the subject, optical gain in materials with indirect transitions was assessed recently based on a simple twolevel model. ${ }^{22)}$

In this paper, we present a considerably more detailed theoretical treatment of optical gain at bandgap energy in indirect bandgap semiconductors, including the calculation of the optical gain in bulk crystalline silicon versus temperature and the derivation of the rate equations of electron, photon, and phonon involved. In $\$ 2$, an analytical expression for optical gain via phonon-assisted optical transitions in indirect bandgap semiconductors which takes into account the structures of the conduction and valence band band-edges is presented. We obtain the conditions required for population inversion in indirect bandgap semiconductors. The rate equations and the steady-state solutions for electron, photon and phonon involved are presented in $\S 3$. In $\$ 4$, calculations of optical gain and free carrier absorption in bulk crystalline silicon at room and low temperatures are provided. The optical gain is shown to be greater than the free carrier absorption at the temperature below $23 \mathrm{~K}$. Finally, discussions and conclusions are provided in $\S 5$ and $\S 6$, respectively.

\section{Optical Gain of Quantum Transition Involving Photons and Phonons}

Figure 1 shows a schematic diagram of optical emission and absorption in indirect bandgap semiconductors such as silicon. In order for the crystal momentum to be conserved,

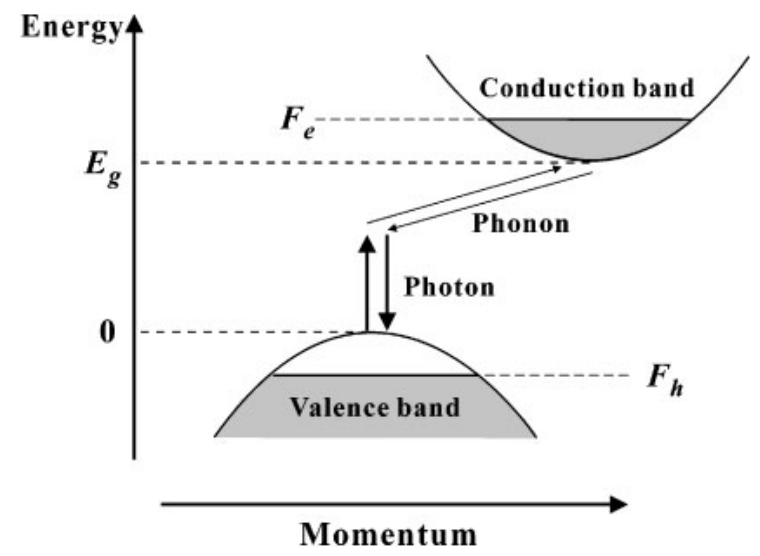

Fig. 1. Schematic diagram of phonon-assisted optical transitions in indirect bandgap semiconductors. 
it is necessary that phonons participate in the optical transitions between the conduction and valence band bandedges. The spectral peak of the spontaneous emission spectrum is known to be slightly below the bandgap energy of silicon, ${ }^{16-19)}$ which means that the optical emission is assisted by emission of a phonon, as depicted in Fig. 1. On the other hand, optical absorption at this photon energy is assisted by the absorption of a phonon, also depicted in Fig. 1. As shown in Appendix A, the theoretical analysis has arrived at an expression for optical gain $g(\hbar \omega)$ via phononassisted optical transitions in indirect bandgap semiconductors such as silicon as follows

$$
\begin{aligned}
g(\hbar \omega)= & \frac{h^{3} c^{2}}{8 \pi n^{2}(\hbar \omega)^{2}} R_{\mathrm{sp}}(\hbar \omega) \\
& \times\left[1-\frac{n_{\mathrm{q}}}{n_{\mathrm{q}}+1} \cdot \exp \left(\frac{\hbar \omega+\hbar \Omega-\Delta F}{k_{\mathrm{B}} T}\right)\right]
\end{aligned}
$$

where $R_{\mathrm{sp}}(\hbar \omega)$ is the spontaneous emission rate, $n_{\mathrm{q}}$ is the phonon occupation number, $\hbar \omega$ and $\hbar \Omega$ are the photon and phonon energies, $\hbar$ is the reduced Planck constant, $c$ is the velocity of light in free space, $n$ is the refractive index, $\Delta F$ is the difference of the quasi-Fermi levels for electrons and holes, $k_{\mathrm{B}}$ is the Boltzmann constant, and $T$ is the temperature. From eq. (1) we see that optical gain can take place if the quantity in braces is positive, i.e.,

$$
\frac{n_{\mathrm{q}}+1}{n_{\mathrm{q}}}>\exp \left(\frac{\hbar \omega+\hbar \Omega-\Delta F}{k_{\mathrm{B}} T}\right) .
$$

Equation (2) indicates that for population inversion or optical gain to occur, the populations of phonons, electrons, and holes must satisfy a certain condition. Note that the term in the left hand side of eq. (2) is the ratio of the phonon emission rate to the phonon absorption rate. ${ }^{23)}$ The term $(\hbar \omega+\hbar \Omega)$ in the right hand side is the energy of the system after stimulated emission of a photon and a phonon, and $\Delta F$ is the energy of the system before stimulated emission. Thus eq. (2) states that for optical gain to occur in the phonon-assisted optical transitions, the ratio of the phonon emission rate to the phonon absorption rate must be greater than a Boltzmann factor determined by the energy difference of the system before and after stimulated emission. We see that

$$
\begin{array}{r}
\frac{n_{\mathrm{q}}+1}{n_{\mathrm{q}}}>1>\exp \left(\frac{\hbar \omega+\hbar \Omega-\Delta F}{k_{\mathrm{B}} T}\right) \\
\text { if } \Delta F>\hbar \omega+\hbar \Omega
\end{array}
$$

Thus, if the difference between the quasi-Fermi levels for electrons and holes is greater than the sum of photon and phonon energies, as shown schematically in Fig. 1, the stimulated emission rate will exceed the absorption rate and population inversion occurs.

Figure 2 shows the relationship between the difference between the quasi-Fermi levels $\Delta F$ and the phonon occupation number $n_{\mathrm{q}}$ required for population inversion involving transverse optical (TO) phonon ( $\hbar \Omega=57.8 \mathrm{meV})$ assisted optical transition at photon energy $\hbar \omega=1.07 \mathrm{eV}$ in

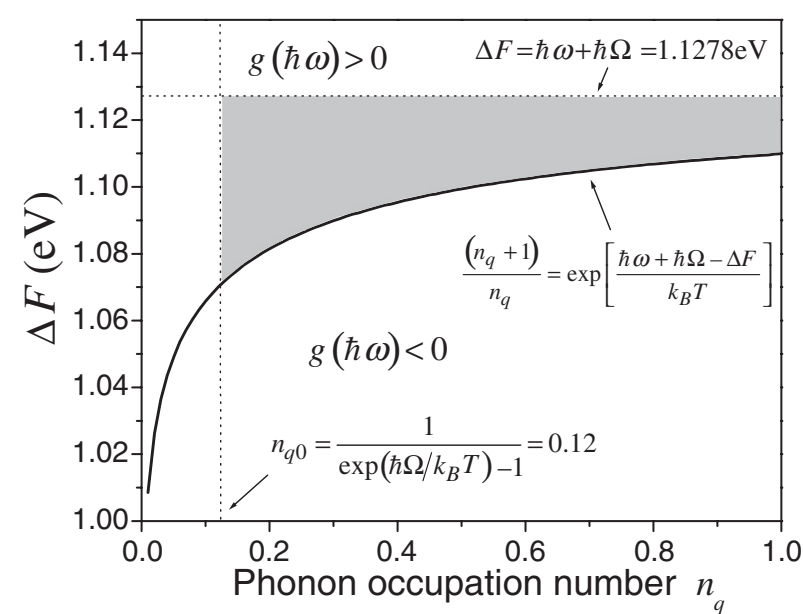

Fig. 2. Relationship between $\Delta F$ and $n_{\mathrm{q}}$ required for population inversion in bulk crystalline silicon at $300 \mathrm{~K}$. The phonon energy $\hbar \Omega$ of $57.8 \mathrm{meV}$ and photon energy $\hbar \omega$ of $1.07 \mathrm{eV}$ were used in the calculation.

crystalline silicon at $300 \mathrm{~K}$. The solid curve in Fig. 2 depicts the condition and the region for population inversion based on eq. (2). The phonon occupation number at thermal equilibrium $\left(n_{\mathrm{q} 0}=0.12\right)$ and the condition $\Delta F=\hbar \omega+$ $\hbar \Omega=1.1278 \mathrm{eV}$ are indicated in dotted lines, respectively. The region above the solid curve in Fig. 2 is the positive optical gain region $[g(\hbar \omega)>0]$, while the region below the solid curve is the absorption region $[g(\hbar \omega)<0]$. Since the energy $\hbar \omega+\hbar \Omega$ is approximately equal to bandgap energy $E_{\mathrm{g}}$, the condition $\Delta F>\hbar \omega+\hbar \Omega \approx E_{\mathrm{g}}$ corresponds to the situation of electronic population inversion. ${ }^{22)}$ It should be noted in Fig. 2 that there exists a region (the shaded area) where population inversion can occur even though $\Delta F<$ $\hbar \omega+\hbar \Omega \approx E_{\mathrm{g}}$. This indicates that the positive optical gain $[g(\hbar \omega)>0]$ can occurs without an electronic population inversion. $^{22)}$

Since a phonon is emitted during the emission of a photon, as shown schematically in Fig. 1, a net stimulated emission of phonons will occur when the population inversion is reached. The condition for the net stimulated phonon emission with energy $\hbar \Omega$ resulting from the net stimulated emission of photons with energy $\hbar \omega$ is the same as eq. (2). In other words, the net stimulated emissions of photons and phonons in indirect bandgap semiconductors take place simultaneously when the condition set by eq. (2) is satisfied. The increase in the phonon population due to the net stimulated phonon emission results in a deviation of phonon occupation number $n_{\mathrm{q}}$ from its value at thermal equilibrium. Figure 2 shows that as the phonon occupation number $n_{\mathrm{q}}$ increases from the value at thermal equilibrium $n_{\mathrm{q} 0}$, a larger value of $\Delta F$ is required to facilitate population inversion.

Similarly, following the procedures similar to those presented in Appendix A, we obtain the expressions for the optical gain associated with emission of multi-phonons as follows

$$
g_{N}(\hbar \omega)=\frac{h^{3} c^{2}}{8 \pi n^{2}(\hbar \omega)^{2}} R_{\mathrm{sp} N}(\hbar \omega) \cdot\left[1-\prod_{i=1}^{N} \frac{n_{\mathrm{q} i}}{n_{\mathrm{q} i}+1} \cdot \exp \left(\frac{\hbar \omega+\hbar \Omega_{1}+\hbar \Omega_{2}+\cdots+\hbar \Omega_{N}-\Delta F}{k_{\mathrm{B}} T}\right)\right]
$$


where $N=1,2,3 \ldots ; g_{N}(\hbar \omega)$ is the optical gain coefficient associated with emission of $N$ phonons; $n_{\mathrm{q} i}$ is the occupation number of the $i$ th phonon mode and $\hbar \Omega_{i}$ is the corresponding energy. Finally, $R_{\mathrm{spN} N}(\hbar \omega)$ is the spontaneous emission rate associated with emission of $N$ phonons. Equation (4) shows that the condition for achieving population inversion associated with $N$-phonon emission is

$$
\prod_{i=1}^{N} \frac{n_{\mathrm{q} i}+1}{n_{\mathrm{q} i}}>\exp \left(\frac{\hbar \omega+\hbar \Omega_{1}+\hbar \Omega_{2}+\cdots+\hbar \Omega_{N}-\Delta F}{k_{\mathrm{B}} T}\right)
$$

We see that

$$
\prod_{i=1}^{N} \frac{n_{\mathrm{q} i}+1}{n_{\mathrm{q} i}}>1>\exp \left(\frac{\hbar \omega+\hbar \Omega_{1}+\hbar \Omega_{2}+\cdots+\hbar \Omega_{N}-\Delta F}{k_{\mathrm{B}} T}\right) \text { if } \Delta F>\hbar \omega+\sum_{i=1}^{N} \hbar \Omega_{i} \approx E_{\mathrm{g}} .
$$

Therefore, once the difference between the quasi-Fermi levels $\Delta F$ is greater than the sum of photon and phonon energies, which is approximately equal to the bandgap energy $E_{\mathrm{g}}$, the population inversions associated with emission of $1,2, \ldots, N$ phonons can take place.

\section{Rate Equations and Co-Stimulated Emissions of Photons and Phonons}

In this section, we present the rate equations for the single-mode photon, phonon and carrier in indirect bandgap semiconductors and, then, determine their steady-state solutions. The spontaneous emission rate $R_{\mathrm{sp}}(\hbar \omega)$, stimulated emission rate $R_{\mathrm{st}}(\hbar \omega)$, and absorption rate $R_{\mathrm{ab}}(\hbar \omega)$ of the quantum transition involving a photon with energy $\hbar \omega$ and a phonon with energy $\hbar \Omega$ are derived in Appendices A and B. They are given as follows

$$
\begin{aligned}
& R_{\mathrm{sp}}(\hbar \omega)=M \cdot\left(n_{\mathrm{q}}+1\right) \cdot N P, \\
& R_{\mathrm{st}}(\hbar \omega)=M \cdot n_{\mathrm{p}}\left(n_{\mathrm{q}}+1\right) \cdot N P, \\
& R_{\mathrm{ab}}(\hbar \omega)=M \cdot n_{\mathrm{p}} n_{\mathrm{q}} \cdot \exp \left(\frac{\hbar \omega+\hbar \Omega-\Delta F}{k_{\mathrm{B}} T}\right) \cdot N P
\end{aligned}
$$

where the proportional constant $M=\pi / 8 \cdot A_{\mathrm{cv}}\left(\hbar \omega+\hbar \Omega-E_{\mathrm{g}}\right)^{2} \exp \left[-\left(\hbar \omega+\hbar \Omega-E_{\mathrm{g}}\right) / k_{\mathrm{B}} T\right]$, in which $A_{\mathrm{cv}}$ is the spontaneous emission coefficient, $N$ and $P$ are the electron and hole concentrations, and $n_{\mathrm{p}}$ is the photon occupation number which gives the average number of photons per state with the photon energy $\hbar \omega$. Therefore, the net generation rates for photon and phonon are given by

$$
R_{\mathrm{st}}(\hbar \omega)-R_{\mathrm{ab}}(\hbar \omega)+R_{\mathrm{sp}}(\hbar \omega)=M \cdot\left[\left(n_{\mathrm{p}}+1\right)\left(n_{\mathrm{q}}+1\right)-n_{\mathrm{p}} n_{\mathrm{q}} \exp \left(\frac{\hbar \omega+\hbar \Omega-\Delta F}{k_{\mathrm{B}} T}\right)\right] \cdot N P .
$$

Note that in eq. (8), the term $\left(n_{\mathrm{p}}+1\right)\left(n_{\mathrm{q}}+1\right) \cdot N P$ corresponds to the stimulated and spontaneous emission rate of photon and phonon, while the term $n_{\mathrm{p}} n_{\mathrm{q}} \exp [(\hbar \omega+\hbar \Omega-$ $\left.\Delta F) / k_{\mathrm{B}} T\right] \cdot N P$ represents the absorption rate of photon and phonon.

Now, the rate equations for electron concentration $N$, photon density $N_{\mathrm{p}}$ and phonon density $N_{\mathrm{q}}$ in the quantum transitions with a single-optical mode associated with the photon energy $\hbar \omega$ and a single-phonon mode associated with the phonon energy $\hbar \Omega$ are given as follows

$$
\begin{aligned}
\frac{d N}{d t} & =R_{\mathrm{p}}-R_{\mathrm{st}}(\hbar \omega)+R_{\mathrm{ab}}(\hbar \omega)-R_{\mathrm{sp}}(\hbar \omega)-\frac{N}{\tau_{\mathrm{c}}}, \\
\frac{d N_{\mathrm{p}}}{d t} & =R_{\mathrm{st}}(\hbar \omega)-R_{\mathrm{ab}}(\hbar \omega)+\beta R_{\mathrm{sp}}(\hbar \omega)-\frac{N_{\mathrm{p}}}{\tau_{\mathrm{p}}}, \\
\frac{d N_{\mathrm{q}}}{d t} & =R_{\mathrm{st}}(\hbar \omega)-R_{\mathrm{ab}}(\hbar \omega)+R_{\mathrm{sp}}(\hbar \omega)-\frac{N_{\mathrm{q}}-N_{\mathrm{q} 0}}{\tau_{\mathrm{q}}}
\end{aligned}
$$

where $R_{\mathrm{p}}$ is the pumping rate either by current injection or optical excitation, $\beta$ is spontaneous emission factor which represents the fraction of spontaneous emission entering the optical mode, $N_{\mathrm{q} 0}$ is the phonon density at thermodynamic equilibrium, and $\tau_{\mathrm{c}}, \tau_{\mathrm{p}}$ and $\tau_{\mathrm{q}}$ are the lifetime of carriers, photons and phonons, respectively. Nonradiative ShockleyRead-Hall recombination is the main mechanism that determines the carrier lifetime $\tau_{\mathrm{c}}$. The loss of photon due to the effects such as optical scattering, free carrier absorption, and output coupling of the resonant cavity, can be characterized by the photon lifetime $\tau_{\mathrm{p}}$. The last term in eq. $(9 \mathrm{c})$ represents anharmonic phonon interactions. Due to the anharmonic interactions between phonons, ${ }^{23)}$ the phonons resulting from the phonon-assisted optical transition will decay to other phonon modes. For example, the transverse optical (TO) phonon generated from optical emission in crystalline silicon will decay into the acoustic phonon modes with a typical phonon lifetime in the order of picoseconds. ${ }^{24)}$ Therefore the phonon density $N_{\mathrm{q}}$ will be restored to its thermal equilibrium value $N_{\mathrm{q} 0}$. The photon density $N_{\mathrm{p}}$ and the phonon density $N_{\mathrm{q}}$ in eqs. (9b) and (9c), respectively, are related to the photon and phonon occupation numbers $n_{\mathrm{p}}$ and $n_{\mathrm{q}}$ as follows

$$
\begin{aligned}
& N_{\mathrm{p}}=K_{\mathrm{p}} n_{\mathrm{p}}, \\
& N_{\mathrm{q}}=K_{\mathrm{q}} n_{\mathrm{q}}
\end{aligned}
$$

in which $K_{\mathrm{p}}$ and $K_{\mathrm{q}}$ are the density of states for the singlephoton and single-phonon modes, respectively. Substituting eqs. (7) and (10) into eq. (9) and assuming that the electron concentration $N$ is equal to the hole concentration $P$ due to charge neutrality, we obtain the following coupled equations: 


$$
\begin{aligned}
\frac{d N}{d t}= & R_{\mathrm{p}}-M n_{\mathrm{p}}\left[\left(n_{\mathrm{q}}+1\right)-n_{\mathrm{q}} \exp \left(\frac{\hbar \omega+\hbar \Omega-\Delta F}{k_{\mathrm{B}} T}\right)\right] N^{2} \\
& -M\left(n_{\mathrm{q}}+1\right) N^{2}-\frac{N}{\tau_{\mathrm{c}}}, \\
\frac{d n_{\mathrm{p}}}{d t}= & \frac{M}{K_{\mathrm{p}}} n_{\mathrm{p}}\left[\left(n_{\mathrm{q}}+1\right)-n_{\mathrm{q}} \exp \left(\frac{\hbar \omega+\hbar \Omega-\Delta F}{k_{\mathrm{B}} T}\right)\right] N^{2} \\
& +\beta \frac{M}{K_{\mathrm{p}}}\left(n_{\mathrm{q}}+1\right) N^{2}-\frac{n_{\mathrm{p}}}{\tau_{\mathrm{p}}}, \\
\frac{d n_{\mathrm{q}}}{d t}= & \frac{M}{K_{\mathrm{q}}} n_{\mathrm{p}}\left[\left(n_{\mathrm{q}}+1\right)-n_{\mathrm{q}} \exp \left(\frac{\hbar \omega+\hbar \Omega-\Delta F}{k_{\mathrm{B}} T}\right)\right] N^{2} \\
& +\frac{M}{K_{\mathrm{q}}}\left(n_{\mathrm{q}}+1\right) N^{2}-\frac{n_{\mathrm{q}}-n_{\mathrm{q} 0}}{\tau_{\mathrm{q}}} .
\end{aligned}
$$

Equations (11a)-(11c) are the rate equations for carrier concentration $N$, photon occupation number $n_{\mathrm{p}}$ and phonon occupation number $n_{\mathrm{q}}$, respectively.

We now set $d / d t=0$ in eqs. (11a)-(11c) and solve for the steady state solution of the rate equations. From eq. (11b), the steady state photon occupation number $n_{\mathrm{p}}$ is readily obtained as follows

$$
n_{\mathrm{p}}=\frac{\frac{1}{K_{\mathrm{p}}} \beta M\left(n_{\mathrm{q}}+1\right) N^{2}}{\frac{1}{\tau_{\mathrm{p}}}-\frac{M}{K_{\mathrm{p}}}\left[\left(n_{\mathrm{q}}+1\right)-n_{\mathrm{q}} \exp \left(\frac{\hbar \omega+\hbar \Omega-\Delta F}{k_{\mathrm{B}} T}\right)\right] N^{2}} .
$$

As the denominator in eq. (12) approaches zero, $n_{\mathrm{p}}$ becomes very large. Thus, the following equation

$$
M\left[\left(n_{\mathrm{q}}+1\right)-n_{\mathrm{q}} \exp \left(\frac{\hbar \omega+\hbar \Omega-\Delta F}{k_{\mathrm{B}} T}\right)\right] N^{2}=\frac{K_{\mathrm{p}}}{\tau_{\mathrm{p}}}
$$

is the threshold condition for laser oscillation, meaning that the photon loss of the resonant cavity must be compensated by the optical gain for the on-set of laser oscillation. In the following, we discuss the steady state solutions in two situations: (1) Below threshold, and (2) Above threshold.

\subsection{Below threshold}

Below the threshold condition for laser oscillation, the photon density is low and the net stimulated emission rate $R_{\mathrm{st}}(\hbar \omega)-R_{\mathrm{ab}}(\hbar \omega)$ in the rate equations can be neglected. Then the rate equations become

$$
\begin{aligned}
& \frac{d N}{d t}=R_{\mathrm{p}}-M\left(n_{\mathrm{q}}+1\right) N^{2}-\frac{N}{\tau_{\mathrm{c}}}=0, \\
& \frac{d n_{\mathrm{p}}}{d t}=\beta \frac{M}{K_{\mathrm{p}}}\left(n_{\mathrm{q}}+1\right) N^{2}-\frac{n_{\mathrm{p}}}{\tau_{\mathrm{p}}}=0, \\
& \frac{d n_{\mathrm{q}}}{d t}=\frac{M}{K_{\mathrm{q}}}\left(n_{\mathrm{q}}+1\right) N^{2}-\frac{n_{\mathrm{q}}-n_{\mathrm{q} 0}}{\tau_{\mathrm{q}}}=0 .
\end{aligned}
$$

We shall now determine the typical values of the two terms in eq. (14c) using the typical data shown in Table I. Accordingly, the first term in eq. (14c) is approximately equal to $10^{-4} \times\left(n_{\mathrm{q}}+1\right) \mathrm{s}^{-1}$, while the second term $\left(n_{\mathrm{q}}-\right.$ $\left.n_{\mathrm{q} 0}\right) / \tau_{\mathrm{q}} \approx 10^{12} \times\left(n_{\mathrm{q}}-n_{\mathrm{q} 0}\right) \mathrm{s}^{-1}$. Consequently, the following approximation is valid

$$
n_{\mathrm{q}} \approx n_{\mathrm{q} 0}
$$

Table I. Typical values of relevant parameters.

\begin{tabular}{llc}
\hline \multicolumn{1}{c}{ Parameter } & Typical value & Reference \\
\hline$R_{\mathrm{p}}\left(\mathrm{cm}^{-3} \mathrm{~s}^{-1}\right)$ & $10^{21}-10^{25}$ & 34 \\
$M\left(\mathrm{~cm}^{3} \mathrm{~s}^{-1}\right)$ & $10^{-15}$ & 41,42 \\
$N\left(\mathrm{~cm}^{-3}\right)$ & $10^{15}-10^{20}$ & 41,42 \\
$K_{\mathrm{p}}\left(\mathrm{cm}^{-3}\right)$ & $10^{11}$ & 34 \\
$K_{\mathrm{q}}\left(\mathrm{cm}^{-3}\right)$ & $10^{23}$ & 23 \\
$\tau_{\mathrm{c}}\left(\mathrm{s}^{-1}\right)$ & $10^{-6}$ & 42 \\
$\tau_{\mathrm{p}}\left(\mathrm{s}^{-1}\right)$ & $10^{-12}$ & 34 \\
$\tau_{\mathrm{q}}\left(\mathrm{s}^{-1}\right)$ & $10^{-12}$ & 24 \\
$n_{\mathrm{q} 0}$ & 0.12 & 23,36 \\
\hline
\end{tabular}

Substituting eq. (15) into eq. (14a) we obtain the following solution for the carrier concentration

$$
N=\frac{-\frac{1}{\tau_{\mathrm{c}}}+\sqrt{\frac{1}{\tau_{\mathrm{c}}^{2}}+4 M\left(n_{\mathrm{q} 0}+1\right) R_{\mathrm{p}}}}{2 M\left(n_{\mathrm{q} 0}+1\right)} .
$$

Again, using the typical values shown in Table I, we see that $1 / \tau_{\mathrm{c}}^{2}$ is much larger than $4 M\left(n_{\mathrm{q} 0}+1\right) R_{\mathrm{p}}$. Thus

$$
N \approx \frac{-\frac{1}{\tau_{\mathrm{c}}}+\frac{1}{\tau_{\mathrm{c}}}\left(1+\frac{1}{2} 4 M \tau_{\mathrm{c}}^{2}\left(n_{\mathrm{q} 0}+1\right) R_{\mathrm{p}}\right)}{2 M\left(n_{\mathrm{q} 0}+1\right)}=\tau_{\mathrm{c}} R_{\mathrm{p}} .
$$

Finally, substituting eqs. (15) and (17) into eq. (14b), the photon density $N_{\mathrm{p}}$ is obtained

$$
N_{\mathrm{p}} \approx \beta \tau_{\mathrm{p}} M \tau_{\mathrm{c}}^{2}\left(n_{\mathrm{q} 0}+1\right) R_{\mathrm{p}}^{2} .
$$

In summary, at below threshold condition the carrier concentration $N$ increases linearly with the pumping rate $R_{\mathrm{p}}$, the phonon occupation number $n_{\mathrm{q}}$ remains close to its thermal equilibrium value $n_{\mathrm{q} 0}$, and the photon density $N_{\mathrm{p}}$ increases with the square of the pumping rate $R_{\mathrm{p}}$.

\subsection{Above threshold}

Equation (3) shows that $\Delta F>\hbar \omega+\hbar \Omega$ is the necessary condition for the occurrence of population inversion. It is usually the case that $\Delta F \gg \hbar \omega+\hbar \Omega$ when the threshold condition eq. (13) is reached. As a result, the factor $\exp \left[(\hbar \omega+\hbar \Omega-\Delta F) / k_{\mathrm{B}} T\right] \ll 1$ can be neglected and the threshold condition eq. (13) is simplified as follows:

$$
M\left(n_{\mathrm{q}}+1\right) N^{2}=\frac{K_{\mathrm{p}}}{\tau_{\mathrm{p}}} .
$$

Thus the rate equations eq. (11) are reduced to the following forms

$$
\begin{aligned}
& \frac{d N}{d t}=R_{\mathrm{p}}-M n_{\mathrm{p}}\left(n_{\mathrm{q}}+1\right) N^{2}-M\left(n_{\mathrm{q}}+1\right) N^{2}-\frac{N}{\tau_{\mathrm{c}}}, \\
& \frac{d n_{\mathrm{p}}}{d t}=\frac{M}{K_{\mathrm{p}}} n_{\mathrm{p}}\left(n_{\mathrm{q}}+1\right) N^{2}+\beta \frac{M}{K_{\mathrm{p}}}\left(n_{\mathrm{q}}+1\right) N^{2}-\frac{n_{\mathrm{p}}}{\tau_{\mathrm{p}}} \\
& \frac{d n_{\mathrm{q}}}{d t}=\frac{M}{K_{\mathrm{q}}} n_{\mathrm{p}}\left(n_{\mathrm{q}}+1\right) N^{2}+\frac{M}{K_{\mathrm{q}}}\left(n_{\mathrm{q}}+1\right) N^{2}-\frac{n_{\mathrm{q}}-n_{\mathrm{q} 0}}{\tau_{\mathrm{q}}} .
\end{aligned}
$$


Substituting the threshold condition of eq. (19) into eqs. (20a) and (20c) and seting $d / d t=0$, the following equation is obtained

$$
n_{\mathrm{q}}^{3}+\left(1-2 A_{1}\right) n_{\mathrm{q}}^{2}+\left(A_{1}^{2}-2 A_{1}\right) n_{\mathrm{q}}+\left(A_{1}^{2}-A_{2}\right)=0
$$

where

$$
A_{1}=n_{\mathrm{q} 0}+\frac{\tau_{\mathrm{q}} R_{\mathrm{p}}}{K_{\mathrm{q}}}, \quad A_{2}=\frac{K_{\mathrm{p}} \tau_{\mathrm{q}}^{2}}{\tau_{\mathrm{p}} M \tau_{\mathrm{c}}^{2} K_{\mathrm{q}}^{2}} .
$$

Using the typical data listed in Table I, the value of $A_{1}^{2}$ is found to be much greater than $A_{2}$, so the $A_{2}$ term in eq. (21) can be neglected. Thus the corresponding steady state solution for phonon occupation number is obtained as follows

$$
n_{\mathrm{q}} \approx A_{1}=n_{\mathrm{q} 0}+\frac{\tau_{\mathrm{q}}}{K_{\mathrm{q}}} R_{\mathrm{p}}
$$

The phonon occupation number $n_{\mathrm{q}}$ is seen to increase linearly with the pumping rate $R_{\mathrm{p}}$. For convenience, using eq. (10b), eq. (22a) is now written in the following form

$$
N_{\mathrm{q}} \approx N_{\mathrm{q} 0}+\tau_{\mathrm{q}} R_{\mathrm{p}} \text {. }
$$

After substituting eq. (22a) into the laser oscillation threshold condition of eq. (19), the following carrier concentration $N$ at steady state is obtained,

$$
N \approx \sqrt{\frac{K_{\mathrm{p}}}{M \tau_{\mathrm{p}}\left(n_{\mathrm{q} 0}+1+\frac{\tau_{\mathrm{q}}}{K_{\mathrm{q}}} R_{\mathrm{p}}\right)}}
$$

Again, using the typical values of parameters listed in Table I, it is seen that the situation $\tau_{\mathrm{q}} R_{\mathrm{p}} / K_{\mathrm{q}} \ll\left(n_{\mathrm{q} 0}+1\right)$ holds. Thus, the following approximate carrier concentration $N$ is obtained

$$
N \approx \sqrt{\frac{K_{\mathrm{p}}}{M \tau_{\mathrm{p}}\left(n_{\mathrm{q} 0}+1\right)}} \equiv N_{\mathrm{th}} .
$$

Equation (23b) shows that the carrier concentration remains nearly constant at $N_{\text {th }}$. Furthermore, from eq. (20a) the solution for photon density $N_{\mathrm{p}}$ is then given as follows

$$
N_{\mathrm{p}} \approx \tau_{\mathrm{p}}\left[R_{\mathrm{p}}-\left(M\left(n_{\mathrm{q} 0}+1\right) N_{\mathrm{th}}^{2}+\frac{N_{\mathrm{th}}}{\tau_{\mathrm{c}}}\right)\right]=\tau_{\mathrm{p}}\left(R_{\mathrm{p}}-R_{\mathrm{pth}}\right)
$$

where

$$
R_{\mathrm{pth}}=M\left(n_{\mathrm{q} 0}+1\right) N_{\mathrm{th}}^{2}+\frac{N_{\mathrm{th}}}{\tau_{\mathrm{c}}} .
$$

is the pumping rate at threshold.

As described above, although the phonon occupation number $n_{\mathrm{q}}$ increases linearly with the pumping rate $R_{\mathrm{p}}$, the amount of increase is small and so remains very close to its thermodynamic equilibrium value $n_{\mathrm{q} 0}$. Thus the expressions for optical gain associated with emission of one phonon and $N$ phonons, i.e., eqs. (1) and (4), reduce to eqs. (26a) and (26b), respectively:

$$
g_{1}(\hbar \omega)=\frac{h^{3} c^{2}}{8 \pi n^{2}(\hbar \omega)^{2}} R_{\mathrm{sp}}(\hbar \omega) \cdot\left[1-\exp \left(\frac{\hbar \omega-\Delta F}{k_{\mathrm{B}} T}\right)\right],
$$

$$
g_{N}(\hbar \omega)=\frac{h^{3} c^{2}}{8 \pi n^{2}(\hbar \omega)^{2}} R_{\mathrm{sp} N}(\hbar \omega) \cdot\left[1-\exp \left(\frac{\hbar \omega-\Delta F}{k_{\mathrm{B}} T}\right)\right],
$$

and

$$
g(\hbar \omega)>0 \text { if } \Delta F>\hbar \omega .
$$

In comparison with eqs. (1), (2), and (4), it is seen that the factors involving phonons, such as $n_{\mathrm{q}}$ and $\hbar \Omega$, disappear in eqs. (26a), (26b), and (27) as a result of $n_{\mathrm{q}} \approx n_{\mathrm{q} 0}$. This means that even though it is necessary that phonons participate in the optical transitions for the conservation of crystal momentum, the population of phonon does not influence the magnitude of optical gain significantly since $n_{\mathrm{q}}$ is close to $n_{\mathrm{q} 0}$ in practice. Equations (26a) and (26b) take the same form as the optical gain in direct bandgap semiconductors, and eq. (27) is also similar to the BernardDuraffourg condition for the occurrence of population inversion in direct bandgap semiconductors. ${ }^{25)}$ Thus, it is seen that population inversion can be accomplished in indirect bandgap semiconductors provided that the difference between the quasi-Fermi levels for electrons and holes, $\Delta F$, is greater than the photon energy. It should be noted that for the direct bandgap semiconductors, since the energy of photon emitted is equal to the bandgap energy, $\Delta F$ has to be greater than the bandgap energy for the achievement of population inversion. However, as shown in Figs. 1 and 2, for the indirect bandgap semiconductors the energy of photon emitted is slightly smaller than the bandgap energy, it is not necessary for $\Delta F$ to be greater than the bandgap energy for the occurrence of population inversion. This is the difference for the condition of population inversion between the direct and indirect bandgap semiconductors.

Equations (26a) and (26b) show that the signs of optical gain coefficients $g(\hbar \omega)$ and $g_{N}(\hbar \omega)$ are determined by the difference between the quasi-Fermi levels $(\Delta F)$, and their magnitudes are proportional to the spontaneous emission rate $R_{\mathrm{sp}}(\hbar \omega)$ and $R_{\mathrm{sp} N}(\hbar \omega)$. Also, as indicated in eq. (A.7), the magnitude of $\Delta F$ strongly influences the spontaneous emission rate $R_{\mathrm{sp}}(\hbar \omega)$. A PN junction is well-known to be a convenient way for injection of electrons and holes with adequate concentrations to achieve the condition for population inversion represented by eq. (27). The gain coefficient can be enhanced by increasing the spontaneous emission rate $R_{\mathrm{sp}}(\hbar \omega)$. Furthermore, it has been well recognized that the spatial confinement caused by the carrier localization structures leads to an increase in the spontaneous emission rate and, thus, the optical gain coefficient. Consequently, a PN junction with carrier localization structures such as a nanostructured silicon $\mathrm{PN}$ junction $\operatorname{diode}^{20)}$ is proposed for this purpose.

In summary, the steady state phonon density $N_{\mathrm{q}}$, carrier concentration $N$, and the photon density $N_{\mathrm{p}}$ are given by eqs. (22b), (23b), and (24), respectively. When the threshold condition is reached, while the carrier concentration remains approximately at $N_{\mathrm{th}}$, both the photon and phonon densities increase linearly with the pumping rate $R_{\mathrm{p}}$.

It is of interest to depict the results for above threshold, i.e., eqs. (22b), (23b), and (24), with those for below threshold, namely, eqs. (15), (17), and (18), as shown in Fig. 3. Below the threshold, the photon density $N_{\mathrm{p}}$ increases 

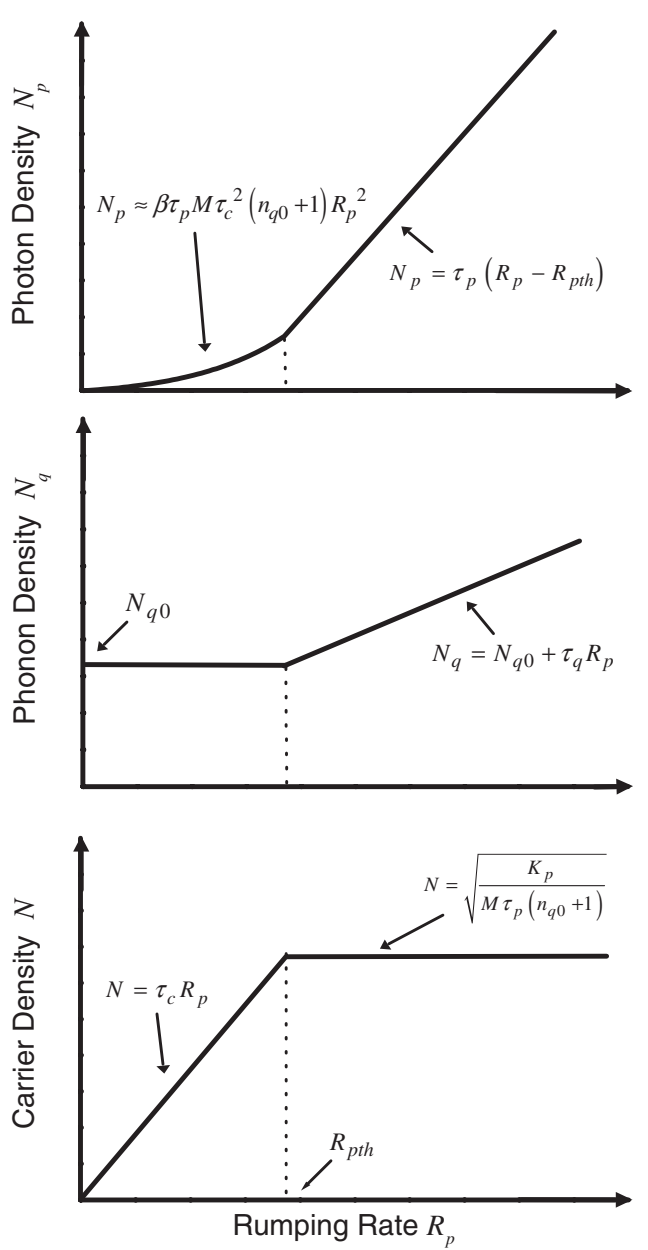

Fig. 3. Phonon, photon, and carrier densities versus the pumping rate below and above threshold.

rather slowly. However, once the threshold is reached, the photon density $N_{\mathrm{p}}$ increases rapidly and linearly with the pumping rate $R_{\mathrm{p}}$. As to the phonon, its density $N_{\mathrm{q}}$ remains practically constant before threshold, but increases linearly with the pumping rate $R_{\mathrm{p}}$ once the threshold is reached. Both the photon and phonon densities grow rapidly after the threshold, which indicates the co-stimulated emission of photons and phonons. On the other hand, the carrier concentration $N$ increases linearly with the pumping rate $R_{\mathrm{p}}$ before the threshold is reached, but clamps at its threshold value $N_{\text {th }}$ after the threshold is reached. In other words, the stimulated emission uses up almost all of the carriers generated by the external pumping after the threshold is reached. Any further increase in the pumping rate $R_{\mathrm{p}}$ does not increase the carrier concentration $N$ but contributes to the increase in photon and phonon densities.

\section{Temperature Dependence of Optical Gain and Free Carrier Absorption}

Equation (27) indicates that population inversion in indirect bandgap semiconductors can occur provided that $\Delta F>\hbar \omega$. However, in order to achieve optical amplification, and eventually lasing, the magnitude of optical gain has to be large enough to overcome the optical losses resulting from the silicon itself and the optical cavity. A major source for material losses is free carrier absorption. ${ }^{26)} \mathrm{We}$ shall now make an estimate on the magnitudes of gain coefficient and free carrier absorption in bulk crystalline silicon.

Equations (26a) and (26b) show that the magnitude of gain coefficient $g(\hbar \omega)$ is proportional to the spontaneous emission rate $R_{\mathrm{sp}}(\hbar \omega)$. Thus the magnitude of optical gain in bulk crystalline silicon can be estimated based on the spontaneous emission coefficient $A_{\mathrm{cv}}$, which is determined here using the optical absorption data in bulk crystalline silicon. ${ }^{27)}$ Appendix B shows that the absorption coefficient $\alpha_{1}(\hbar \omega)$ associated with the one-phonon assisted optical transition is given by

$$
\alpha_{1}(\hbar \omega)=C_{1} \cdot \frac{1}{(\hbar \omega)^{2}} \cdot \frac{1}{\left[1-\exp \left(-\hbar \Omega_{1} / k_{\mathrm{B}} T\right)\right]} \cdot \frac{\pi}{8}\left(\hbar \omega+\hbar \Omega_{1}-E_{\mathrm{g}}\right)^{2} \cdot \exp \left[-\left(\hbar \omega+\hbar \Omega_{1}\right) / k_{\mathrm{B}} T\right]\left[\exp \left(\hbar \omega / k_{\mathrm{B}} T\right)-1\right]
$$

where $C_{1}$ is a constant which is proportional to the spontaneous emission coefficient $A_{\mathrm{cv}}$ and is independent of temperature. ${ }^{28)}$ Using the optical absorption coefficient associated with one TO phonon $\left(\hbar \Omega_{1}=57.8 \mathrm{meV}\right)$ in bulk crystalline silicon, $\left.{ }^{27}\right)$ the value of $C_{1}$ is estimated to be $2.2 \times 10^{4} \mathrm{~cm}^{-1}$. As shown in Appendix A, by rewriting eq. (26a) in terms of the constant $C_{1}$, the magnitude of optical gain associated with the one-phonon assisted process can be determined using the following equation

$$
\begin{aligned}
g_{1}(\hbar \omega)= & C_{1} \cdot \frac{1}{(\hbar \omega)^{2}} \cdot \frac{1}{\left[1-\exp \left(-\hbar \Omega_{1} / k_{\mathrm{B}} T\right)\right]} \cdot\left\{1-\exp \left[(\hbar \omega-\Delta F) / k_{\mathrm{B}} T\right]\right\} \\
& \times \int_{E_{\mathrm{c}}} \int_{E_{\mathrm{v}}}\left[E_{\mathrm{c}}-\left(E_{\mathrm{g}}-\Delta E_{\mathrm{g}}\right)\right]^{1 / 2}\left(-E_{\mathrm{v}}\right)^{1 / 2} f_{\mathrm{c}}\left(E_{\mathrm{c}}\right)\left[1-f_{\mathrm{v}}\left(E_{\mathrm{v}}\right)\right] \cdot \delta\left(\hbar \omega-E_{\mathrm{c}}+E_{\mathrm{v}}+\hbar \Omega_{1}\right) d E_{\mathrm{v}} d E_{\mathrm{c}}
\end{aligned}
$$

where $f_{\mathrm{c}}$ and $\left(1-f_{\mathrm{v}}\right)$ are the Fermi-Dirac distributions with respective quasi-Fermi levels $F_{\mathrm{e}}$ and $F_{\mathrm{h}}$ for electrons and holes, and the integration variables $E_{\mathrm{c}}$ and $E_{\mathrm{v}}$ are the energies of the conduction band and valence band states, respectively.

The calculated gain spectra of bulk crystalline silicon for various values of $\Delta F$ at $300 \mathrm{~K}$ are plotted in Fig. 4 . In this calculation, the effect of bandgap narrowing $\Delta E_{\mathrm{g}}$ due to high carrier density was taken into account using the following relationship ${ }^{29)}$

$$
\Delta E_{\mathrm{g}}=14 \cdot \ln \left(N / 1.4 \times 10^{17}\right) \quad \mathrm{meV}
$$

where $N$ is the carrier density in $\mathrm{cm}^{-3}$. Also, the relationship between the quasi-Fermi levels, $F_{\mathrm{e}}$ and $F_{\mathrm{h}}$, and the carrier density was calculated using the Joyce-Dixon approximation. ${ }^{30)}$ As shown in Fig. 4, when the value of $\Delta F$ increases, the magnitude of optical gain coefficient is enhanced and the range of photon energy corresponding to positive optical gain also increases. The gain coefficient changes sign to become absorption when the photon energy is greater than 


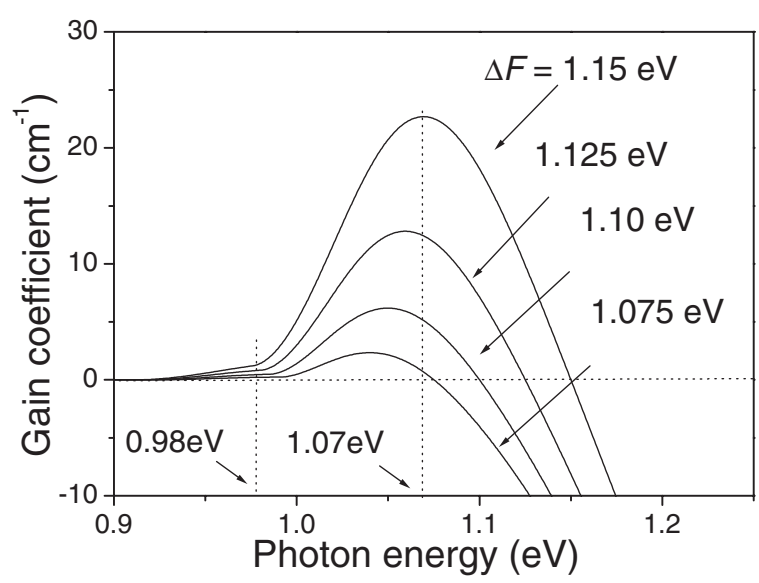

Fig. 4. Calculated gain spectra of bulk crystalline silicon for various values of $\Delta F$ at $300 \mathrm{~K}$.

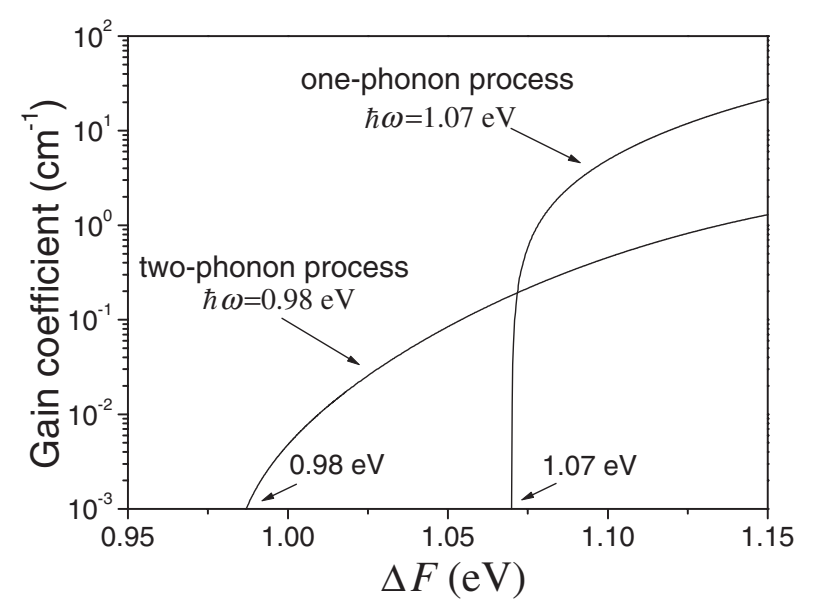

Fig. 5. Calculated gain coefficients of one- and two-phonon-assisted processes in bulk crystalline silicon versus $\Delta F$ at $300 \mathrm{~K}$.

$\Delta F$. The peak in the gain spectra near photon energy $1.07 \mathrm{eV}$ is associated with one TO phonon-assisted radiative recombination, ${ }^{31,32)}$ and the shoulder in the gain spectra around photon energy $0.98 \mathrm{eV}$ is attributed to two-phonon ( $\mathrm{TO}+$ $\mathrm{O}^{\Gamma}$ )-assisted radiative recombination. ${ }^{31,32)}$ According to the measured photoluminescence spectra of bulk crystalline silicon at $26 \mathrm{~K}^{31)}$ the ratio of the spontaneous emission rate of the two-phonon process $R_{\mathrm{sp} 2}(\hbar \omega)$ to the one-phonon process $R_{\mathrm{sp} 1}(\hbar \omega)$ is 0.07 , and this value was adopted in this calculation.

Figure 5 shows the plot of gain coefficients (in logarithmic scale) of bulk crystalline silicon versus the difference between the quasi-Fermi levels $\Delta F$ for one- and twophonon-assisted processes at $300 \mathrm{~K}$. The optical gains are negative for both the one- and two-phonon processes when $\Delta F<0.98 \mathrm{eV}$. For the range $0.98<\Delta F<1.07 \mathrm{eV}$, positive optical gain only occurs for the two-phonon process while the one-phonon process still results in absorption. Population inversion for both one- and two-phonon processes takes place simultaneously when $\Delta F>1.07 \mathrm{eV}$. Figure 5 indicates that two-phonon assisted radiative recombination requires a smaller value of $\Delta F$ to facilitate population inversion, as compared to the one-phonon process.

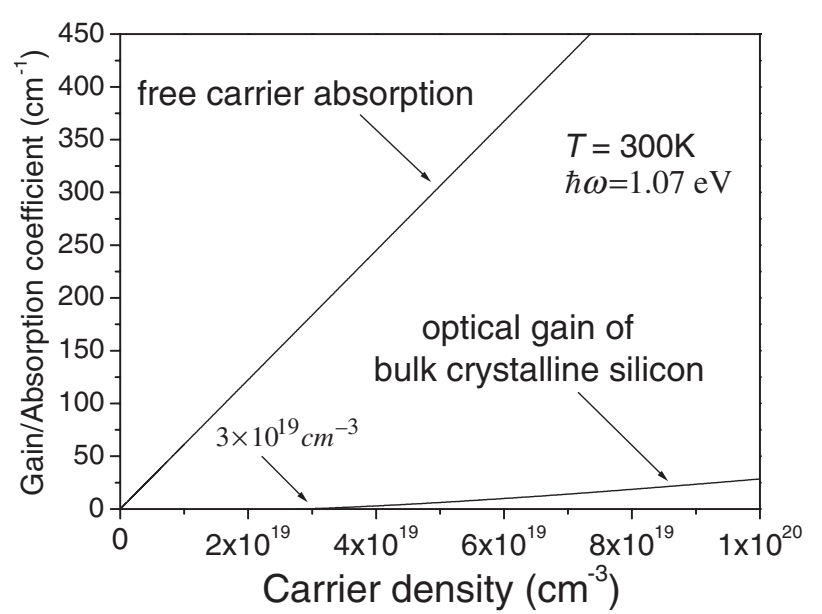

Fig. 6. Calculated optical gain and free carrier absorption coefficients in bulk crystalline silicon versus the carrier density at photon energy $\hbar \omega=$ $1.07 \mathrm{eV}$ at $300 \mathrm{~K}$.

Figures 4 and 5 show that the maximum gain coefficient in bulk crystalline silicon can be as large as $22.5 \mathrm{~cm}^{-1}$ when $\Delta F=1.15 \mathrm{eV}$. However, optical amplification in bulk crystalline silicon around the bandgap energy $E_{\mathrm{g}}$ has never been observed at room temperature. This fact may be attributed to the excessive free carrier absorption. ${ }^{26)}$ The magnitude of free carrier absorption $\alpha_{\mathrm{fc}}$ at room temperature in units of $\mathrm{cm}^{-1}$ is given by ${ }^{28,33)}$

$$
\alpha_{\mathrm{fc}}=\left(1.01 \times 10^{-20} N+0.51 \times 10^{-20} P\right) \lambda^{2} T
$$

where $N$ and $P$ are again the carrier densities in units of $\mathrm{cm}^{-3}$ for electrons and holes, and $\lambda$ is the wavelength in $\mu \mathrm{m}$, and $T$ is the temperature in K. The plots in Fig. 6 show the magnitudes of optical gain and free carrier absorption in bulk crystalline silicon versus the carrier density at photon energy $\hbar \omega$ of $1.07 \mathrm{eV}$ at $300 \mathrm{~K}$. In the calculation, the electron concentration $N$ is assumed to be equal to the hole concentration $P$ due to charge neutrality. The gain coefficient increases linearly with the carrier density, and the transparent carrier density is $3 \times 10^{19} \mathrm{~cm}^{-3}$ in bulk crystalline silicon, which is about one order of magnitude greater than that in bulk GaAs. ${ }^{34)}$ Note that the transparent carrier density is defined as the carrier density where stimulated emission rate $R_{\mathrm{st}}(\hbar \omega)$ equals absorption rate $R_{\mathrm{ab}}(\hbar \omega){ }^{34)}$ Figure 6 clearly demonstrates that the free carrier absorption is larger than the optical gain in bulk crystalline silicon. This conclusion is identical to that of Dumke. ${ }^{26)}$ As a result, even though the condition for population inversion eq. (27) is met, it is not possible to accomplish optical gain or optical amplification in bulk crystalline silicon at room temperature.

In Fig. 7, we show the calculated gain spectra of bulk crystalline silicon for the carrier concentrations of $8.8 \times$ $10^{19} \mathrm{~cm}^{-3}$ at the temperatures of 10,150 , and $300 \mathrm{~K}$. In this calculation, the temperature dependence of the bandgap energy is described by the Varshni's equation ${ }^{35}$ )

$$
E_{\mathrm{g}}(T)=E_{\mathrm{g}}(0)-\frac{A T^{2}}{T+B}
$$

where $E_{\mathrm{g}}(0)=1.17 \mathrm{eV}, A=4.9 \times 10^{-4} \mathrm{eV} / \mathrm{K}$, and $B=$ $655 \mathrm{~K}$. We use the inverse first-order Sommerfeld approximation to calculate the relationship between the quasi- 


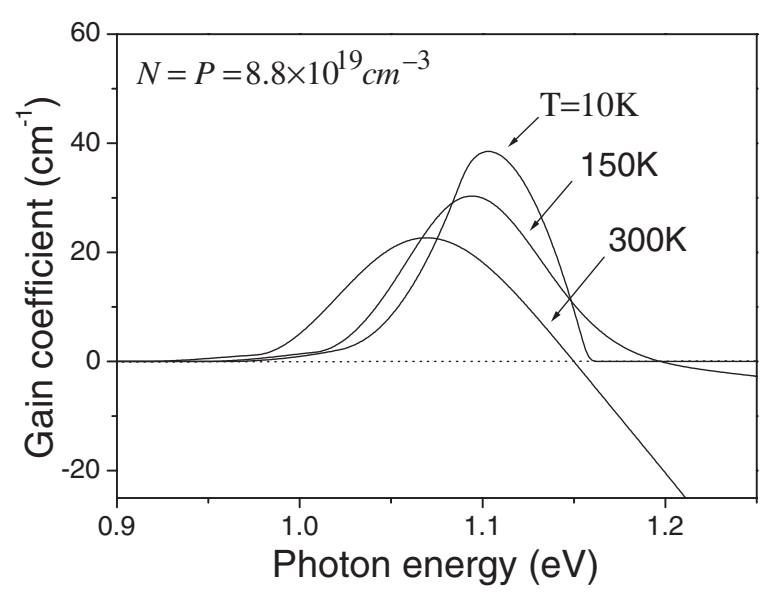

Fig. 7. Calculated gain spectra of bulk crystalline silicon for the carrier concentration of $8.8 \times 10^{19} \mathrm{~cm}^{-3}$ at the temperatures of 10,150 , and $300 \mathrm{~K}$.

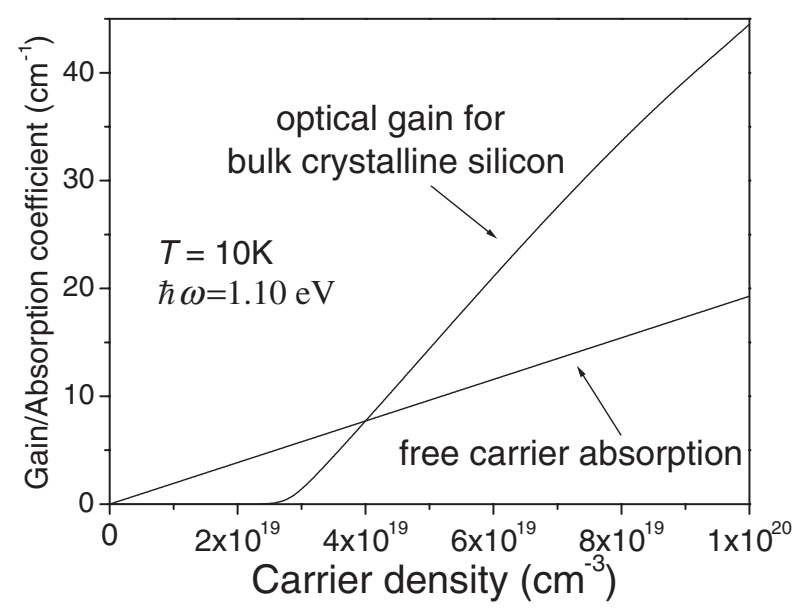

Fig. 8. Calculated optical gain and the free carrier absorption coefficients vs the carrier density in bulk crystalline silicon at photon energy $\hbar \omega=$ $1.10 \mathrm{eV}$ at $10 \mathrm{~K}$.

Fermi levels and the carrier density when the temperature is below $100 \mathrm{~K}^{34)}$ Figure 7 shows that the gain spectra is blueshifted due to the increase in the bandgap energy when the temperature is lowered. The magnitude of optical gain is also shown to increase with decreasing temperature. On the other hand, it should be noted from eq. (31) that as the temperature decreases, the magnitude of free carrier absorption also decreases. Such a temperature dependence is associated with the acoustic phonon scattering. ${ }^{33)}$ Thus it suggests that the optical gain may be larger than the free carrier absorption at a sufficiently low temperature. For this reason we calculate the magnitude of optical gain and free carrier absorption at low temperature. The calculation shows that the optical gain is greater than the free carrier absorption when the temperature is below $23 \mathrm{~K}$. For example, Fig. 8 shows the calculated gain coefficient and free carrier absorption in the bulk crystalline silicon versus the carrier density at photon energy $\hbar \omega=1.1 \mathrm{eV}$ at the temperature of $10 \mathrm{~K}$. It is seen that at the temperature of $10 \mathrm{~K}$, the optical gain greatly exceeds the free carrier absorption when the carrier density is greater than $4 \times 10^{19} \mathrm{~cm}^{-3}$. Therefore, the optical amplification, as well as the co-stimulated emissions of photons and phonons, can be achieved in bulk crystalline silicon at the low temperature below $23 \mathrm{~K}$.

\section{Discussion}

The recent paper by Trupke et al. presented a reassessment of optical gain in indirect bandgap semiconductors based on a simple two-level model. ${ }^{22)}$ The paper points out that the magnitude of optical gain can be orders of magnitude greater than the absorption coefficient and, thus, suggests that the possibility of laser operation in bulk crystalline silicon should be readdressed. In comparison with ref. 22, the theoretical treatment presented here is more specific as it takes into account three important aspects: (1) the structures of the conduction and valence band bandedges, (2) the temperature dependence, and (3) the effect of bandgap narrowing. Using the expressions obtained for the optical gain, the gain spectrum in bulk crystalline silicon is calculated and the magnitude of optical gain is obtained. Furthermore, this treatment explicitly shows that the magnitude of the gain coefficient in bulk crystalline silicon is smaller than the free carrier absorption at room temperature. Thus optical amplification is not possible for bulk crystalline silicon at room temperature. However, at the low temperature below $23 \mathrm{~K}$, the optical gain is shown, for the first time, to be greater than the free carrier absorption. This result indicates that it is possible to achieve optical amplification in bulk crystalline silicon at the temperature below $23 \mathrm{~K}$. In addition, the rate equations and their steady state solutions for electron, photon, and phonon involved in the phonon-assisted optical transitions are obtained. It is shown that once the threshold condition for laser oscillation is reached, co-stimulated emissions of photons and phonons will take place in indirect bandgap semiconductors.

On the other hand it should also be noted that some of the theoretical findings presented in this paper differ from that of Dumke. ${ }^{26)}$ He estimated the magnitude of optical gain in indirect semiconductors and concluded that optical amplification in germanium and silicon is impossible because the optical gain is too small to overcome the free carrier absorption. Dumke's calculations are based on the assumption that the absolute value of maximum optical gain equals the absorption coefficient measured under thermal equilibrium. However, this assumption is questionable for the phonon-assisted optical transitions in indirect bandgap semiconductors. $^{22)}$ As shown in Figs. 4-6, we find that the gain coefficient around the photon energy of $1.07 \mathrm{eV}$ in bulk crystalline silicon at room temperature can be as large as $22.5 \mathrm{~cm}^{-1}$, which is at least two orders of magnitude greater than the absorption coefficient $\left(0.58 \mathrm{~cm}^{-1}\right){ }^{27)}$ Also, as shown in Appendix C, such a large disparity between the optical gain and absorption coefficients results from the involvement of phonons in optical transitions and the effect of bandgap narrowing at high carrier density. At a given photon energy, the stimulated emission of photons is assisted by emission of phonons, while the absorption of photons is also assisted by absorption of phonons. The difference in the phonon emission and absorption rates results in the large disparity between the maximum optical gain and absorption coefficient in bulk crystalline silicon. For a given photon energy, the bandgap narrowing at high carrier density results 
in the increase in the density of conduction and valence band states involved in the optical transition, and thus causes the gain coefficient to increase.

\section{Conclusions}

A theoretical study on optical gain at bandgap energy in indirect bandgap semiconductors, such as silicon, is presented in this paper. The expressions for optical gain associated with one and multi-phonon emission in indirect bandgap semiconductors are obtained. Population inversion can be achieved in indirect bandgap semiconductors when the difference between the quasi-Fermi levels for electrons and holes, $\Delta F$, is greater than the photon energy. It is to be emphasized that two-phonon process requires a lower value of $\Delta F$ to facilitate population inversion than the one-phonon process. In contrast to the direct bandgap semiconductors, population inversion in the indirect bandgap semiconductors can be achieved even if $\Delta F$ is smaller than the bandgap energy.

The rate equations for the single-mode photon, phonon and carrier in indirect bandgap semiconductor are also presented. The condition required for laser oscillation and the steady state solutions below and above threshold are obtained. Below the threshold the photon and phonon densities increase slowly with the pumping rate. However, once the threshold is reached, both the photon and phonon densities increase rapidly and linearly with the pumping rate. The simultaneous increase of the photon and phonon densities can be easily understood since a phonon is emitted during the emission of a photon, a stimulated emission of phonons will take place due to the stimulated emission of photons. Thus co-stimulated emissions of photons and phonons will take place when the threshold condition for laser oscillation is satisfied. As for the carrier concentration, it increases linearly with the pumping rate before the threshold is reached, but remains practically at its threshold value after the threshold is reached.

The temperature dependence of optical gain and free carrier absorption in bulk crystalline silicon is calculated for the first time. It is shown that the optical gain is not sufficiently large to overcome the free carrier absorption at room temperature. Accordingly, it is impossible to observe optical amplification in bulk crystalline silicon at room temperature. However, at the temperature below $23 \mathrm{~K}$, the gain coefficient exceeds the free carrier absorption. It suggests that the co-stimulated emissions of photons and phonons as well as the optical amplification can take place in bulk crystalline silicon at the temperature below $23 \mathrm{~K}$.

\section{Acknowledgement}

This work was initiated at and supported by the Academia Sinica and was subsequently supported in part by the National Science Council, Taiwan, R.O.C under Contract No. NSC94-2112-M-002-019 (M. J. Chen and M. K. Wu), and the Nicholas Foundation Prize, University of California at Irvine, California, U.S.A. (C. S. Tsai).

1) R. A. Soref: Proc. IEEE 81 (1993) 1687.

2) R. A. Soref: Thin Solid Films 294 (1997) 325.

3) O. Boyraz and B. Jalali: Opt. Express 12 (2004) 5269.

4) H. Rong, R. Jones, A. Liu, O. Cohen, D. Hak, A. Fang and
M. Paniccia: Nature 433 (2005) 725

5) See, for example, D. J. Lockwood: Light emission in Silicon: from Physics to Devices (Academic, New York, 1998).

6) L. Pavesi, S. V. Gaponenko and L. Dal Negro: Towards the First Silicon Lasers (Kluwer Academic, Dordrecht, 2003) NATO Science Series.

7) L. Pavesi: Mat. Today 8 (2005) 18.

8) L. Pavesi, L. Dal Negro, C. Mazzoleni, G. Franzo and F. Priolo: Nature 408 (2000) 440.

9) L. Tsybeskov, K. L. Moore, D. G. Hall and P. M. Fauchet: Phys. Rev. B 54 (1996) R8361.

10) Z. H. Lu, D. J. Lockwood and J. M. Baribeau: Nature 378 (1995) 258.

11) J. Ruan, P. M. Fauchet, L. Dal Negro, M. Cazzanelli and L. Pavesi: Appl. Phys. Lett. 83 (2003) 5479.

12) L. T. Canham: Appl. Phys. Lett. 57 (1990) 1046.

13) J. Palm, F. Gan, B. Zheng, J. Michel and L. C. Kimerling: Phys. Rev. B 54 (1996) 17603.

14) P. G. Kik, A. Polman, S. Libertino and S. Coffa: J. Lightwave Technol. 20 (2002) 862.

15) S. G. Cloutier, P. A. Kossyrev and J. Xu: Nat. Mater. 4 (2005) 887.

16) L. Tsybeskov, K. L. Moore, S. P. Duttagupta, K. D. Hirschman, D. G. Hall and P. M. Fauchet: Appl. Phys. Lett. 69 (1996) 3411.

17) W. L. Ng, M. A. Louren, R. M. Gwilliam, S. Ledain, G. Shao and K. P. Homewood: Nature 410 (2001) 192.

18) M. A. Green, J. Zhao, A. Wang, P. J. Reece and M. Gal: Nature 412 (2001) 805.

19) M. J. Chen, J. F. Chang, J. L. Yen, C. S. Tsai, E. Z. Liang, C. F. Lin and C. W. Liu: J. Appl. Phys. 93 (2003) 4253.

20) M. J. Chen, J. L. Yen, J. Y. Li, J. F. Chang, S. C. Tsai and C. S. Tsai: Appl. Phys. Lett. 84 (2004) 2163.

21) H. A. Atwater, G. I. Bourianoff and R. J. Walters: Nat. Mater. 4 (2005) 143.

22) T. Trupke, M. A. Green and P. Würfel: J. Appl. Phys. 93 (2003) 9058.

23) N. W. Ashcroft and N. D. Mermin: Solid State Physics (Brooks Cole, 1976).

24) J. Shah: Ultrafast Spectroscopy of Semiconductors and Semiconductor Nanostructures (Springer-Verlag, Heidelberg, 1999) 2nd ed.

25) M. G. A. Bernard and G. Duraffourg: Phys. Status Solidi 1 (1961) 699.

26) W. P. Dumke: Phys. Rev. 127 (1962) 1559.

27) G. G. Macfarlane, T. P. McLean, J. E. Quarrington and V. Roberts: Phys. Rev. 111 (1958) 1245.

28) J. C. Sturm and C. M. Reaves: IEEE Trans. Electron Devices 39 (1992) 81

29) A. Cuevasa, P. A. Basoreb, G. Giroult-Matlakowski and C. Dubois: J. Appl. Phys. 80 (1996) 3370.

30) W. B. Joyce and R. W. Dixon: Appl. Phys. Lett. 31 (1977) 354.

31) P. J. Dean, J. R. Haynes and W. F. Flood: Phys. Rev. 161 (1967) 711.

32) G. Davies: Phys. Rep. 176 (1989) 83.

33) K. G. Svantesson and N. G. Nilsson: J. Phys. C 12 (1979) 3837.

34) L. A. Coldren and S. W. Corzine: Diode Lasers and Photonic Integrated Circuit (Wiley, New York, 1995).

35) V. Alex, S. Finkbeiner and J. Weber: J. Appl. Phys. 79 (1996) 6943.

36) B. K. Ridley: Quantum Processes in Semiconductors (Oxford University Press, Oxford, 1999) 3rd ed.

37) See, for example, A. E. Siegman: Lasers (University Science Books, Mill Vallet, 1986)

38) A. Yariv: Quantum Electronics (Wiley, New York, 1989) 3rd ed.

39) W. van Roosbroeck and W. Shockley: Phys. Rev. 94 (1954) 1558.

40) H. Schlangenotto, H. Maeder and W. Gerlach: Phys. Status Solidi A 21 (1974) 357.

41) G. Augustine, A. Rohatgi and N. M. Jokerst: IEEE Trans. Electron Devices 39 (1992) 2395.

42) D. K. Schroder: Semiconductor Material and Device Characterization (Wiley, New York, 1998) 2nd ed.

\section{Appendix A}

\section{A.1 Stimulated emission rate $R_{s t}(\hbar \omega)$}

The stimulated emission rate $R_{\mathrm{st}}(\hbar \omega)$ per unit volume of the quantum transition involving the emission of a photon with energy $\hbar \omega$ and a phonon with energy $\hbar \Omega$ is given by $^{23)}$ 


$$
\begin{aligned}
& R_{\mathrm{st}}(\hbar \omega)=B_{\mathrm{cv}} \cdot \rho_{\mathrm{ph}}(\hbar \omega) \\
& \quad \times \int_{E_{\mathrm{c}}} \int_{E_{\mathrm{v}}}\left(n_{\mathrm{q}}+1\right) \cdot D_{\mathrm{c}}\left(E_{\mathrm{c}}\right) D_{\mathrm{v}}\left(E_{\mathrm{v}}\right) f_{\mathrm{c}}\left(E_{\mathrm{c}}\right) \\
& \quad \times\left[1-f_{\mathrm{v}}\left(E_{\mathrm{v}}\right)\right] \cdot \delta\left(\hbar \omega-E_{\mathrm{c}}+E_{\mathrm{v}}+\hbar \Omega\right) d E_{\mathrm{v}} d E_{\mathrm{c}}
\end{aligned}
$$

where $B_{\mathrm{cv}}$ is the stimulated emission coefficient, and $\rho_{\mathrm{ph}}(\hbar \omega)$ is the photon density per energy interval. In eq. $(\mathrm{A} \cdot 1)$, the integration must cover those states in both the conduction and valence bands that may participate in the transition with photon energy $\hbar \omega$ because the momentum in the initial state and final state are not the same. The integral variables $E_{\mathrm{c}}$ and $E_{\mathrm{v}}$ are the energies of the conduction band and valence band states, respectively. The delta function in the integral restricts $E_{\mathrm{c}}$ and $E_{\mathrm{v}}$ to the energy states which contribute to the quantum transitions involving the emission of a photon with energy $\hbar \omega$ and a phonon with energy $\hbar \Omega . D_{\mathrm{c}}\left(E_{\mathrm{c}}\right)$ and $D_{\mathrm{v}}\left(E_{\mathrm{v}}\right)$ are the well-known densities of states for electrons and holes in the conduction and valence bands

$$
\begin{aligned}
D_{\mathrm{c}}\left(E_{\mathrm{c}}\right) & =N_{\mathrm{c}}\left(E_{\mathrm{c}}-E_{\mathrm{g}}\right)^{1 / 2}, \\
N_{\mathrm{c}} & =\left(1 / 2 \pi^{2}\right) \cdot\left(2 m_{\mathrm{e}}^{*} / \hbar^{2}\right)^{3 / 2}, \\
D_{\mathrm{v}}\left(E_{\mathrm{v}}\right) & =N_{\mathrm{v}}\left(-E_{\mathrm{v}}\right)^{1 / 2}, \\
N_{\mathrm{v}} & =\left(1 / 2 \pi^{2}\right) \cdot\left(2 m_{\mathrm{h}}^{*} / \hbar^{2}\right)^{3 / 2}
\end{aligned}
$$

where $\hbar \equiv h / 2 \pi$ is the reduced Planck constant, $h$ is Planck constant, $E_{\mathrm{g}}$ is the indirect bandgap energy, and $m_{\mathrm{e}}^{*}$ and $m_{\mathrm{h}}^{*}$ are the effective masses of electron and hole, respectively. In our calculation, the energy of the top of the valence band is taken as zero, as depicted in Fig. 1. The distribution functions $f_{\mathrm{c}}$ and $\left(1-f_{\mathrm{v}}\right)$ for electrons and holes are given by the Fermi-Dirac distribution with respective quasi-Fermi levels $F_{\mathrm{e}}$ and $F_{\mathrm{h}}$. When the quasi-Fermi levels for electrons and holes locate in the bandgap and far away from the conduction and valence band edges, respectively, the distribution functions $f_{\mathrm{c}}$ and $\left(1-f_{\mathrm{v}}\right)$ may be approximated by the Boltzmann distribution

$$
\begin{gathered}
f_{\mathrm{c}}\left(E_{\mathrm{c}}\right)=\frac{1}{1+\exp \left[\left(E_{\mathrm{c}}-F_{\mathrm{e}}\right) / k_{\mathrm{B}} T\right]} \approx \exp \left[-\left(E_{\mathrm{c}}-F_{\mathrm{e}}\right) / k_{\mathrm{B}} T\right] \quad \text { if }\left(E_{\mathrm{c}}-F_{\mathrm{e}}\right) / k_{\mathrm{B}} T \gg 1, \\
1-f_{\mathrm{v}}\left(E_{\mathrm{v}}\right)=1-\frac{1}{1+\exp \left[\left(E_{\mathrm{v}}-F_{\mathrm{h}}\right) / k_{\mathrm{B}} T\right]}=\frac{1}{1+\exp \left[\left(F_{\mathrm{h}}-E_{\mathrm{v}}\right) / k_{\mathrm{B}} T\right]} \approx \exp \left[-\left(F_{\mathrm{h}}-E_{\mathrm{v}}\right) / k_{\mathrm{B}} T\right] \\
\text { if }\left(F_{\mathrm{h}}-E_{\mathrm{v}}\right) / k_{\mathrm{B}} T \gg 1
\end{gathered}
$$

where $k_{\mathrm{B}}$ is Boltzmann constant and $T$ is absolute temperature.

Finally, the factor $n_{\mathrm{q}}$ in eq. (A.1) is the phonon occupation number, and the probability of phonon emission is proportional to $\left(n_{\mathrm{q}}+1\right){ }^{36)}$ Since the phonons participating in the compensation of the momentum mismatch lie near the Brillouin zone edge, the phonon energy $\hbar \Omega$ can be considered independent of its wavevector. ${ }^{36)}$ At thermodynamic equilibrium, the phonon occupation number $n_{\mathrm{q}}$ becomes $n_{\mathrm{q} 0}$ and is given by Bose-Einstein statistics ${ }^{36)}$

$$
n_{\mathrm{q}}=n_{\mathrm{q} 0}=\frac{1}{\exp \left(\hbar \Omega / k_{\mathrm{B}} T\right)-1}
$$

Thus $n_{\mathrm{q}}$ is also independent of the wavevector as well as $E_{\mathrm{c}}$ and $E_{\mathrm{v}}$. As a result, the $\left(n_{\mathrm{q}}+1\right)$ term may be taken out of the integral in eq. (A.1). Using eqs. (A.2) and (A.3), eq. (A.1) is reduced to

$$
\begin{aligned}
R_{\mathrm{st}}(\hbar \omega)= & B_{\mathrm{cv}} \cdot \rho_{\mathrm{ph}}(\hbar \omega) \cdot\left(n_{\mathrm{q}}+1\right) \cdot N_{\mathrm{c}} N_{\mathrm{v}} \\
& \times \int_{E_{\mathrm{c}}} \int_{E_{\mathrm{v}}}\left(E_{\mathrm{c}}-E_{\mathrm{g}}\right)^{1 / 2}\left(-E_{\mathrm{v}}\right)^{1 / 2} \exp \left[-\left(E_{\mathrm{c}}-E_{\mathrm{v}}-\Delta F\right) / k_{\mathrm{B}} T\right] \cdot \delta\left(\hbar \omega-E_{\mathrm{c}}+E_{\mathrm{v}}+\hbar \Omega\right) d E_{\mathrm{v}} d E_{\mathrm{c}} \\
= & B_{\mathrm{cv}} \cdot \rho_{\mathrm{ph}}(\hbar \omega) \cdot\left(n_{\mathrm{q}}+1\right) \cdot N_{\mathrm{c}} N_{\mathrm{v}} \cdot \exp \left[-(\hbar \omega+\hbar \Omega-\Delta F) / k_{\mathrm{B}} T\right] \\
& \times \int_{E_{\mathrm{g}}}^{\hbar \omega+\hbar \Omega}\left(E_{\mathrm{c}}-E_{\mathrm{g}}\right)^{1 / 2}\left(\hbar \omega-E_{\mathrm{c}}+\hbar \Omega\right)^{1 / 2} d E_{\mathrm{c}} \\
= & B_{\mathrm{cv}} \cdot N_{\mathrm{c}} N_{\mathrm{v}} \cdot \rho_{\mathrm{ph}}(\hbar \omega) \cdot\left(n_{\mathrm{q}}+1\right) \cdot \frac{\pi}{8}\left(\hbar \omega+\hbar \Omega-E_{\mathrm{g}}\right)^{2} \cdot \exp \left[-(\hbar \omega+\hbar \Omega-\Delta F) / k_{\mathrm{B}} T\right]
\end{aligned}
$$

where $\Delta F=F_{\mathrm{e}}-F_{\mathrm{h}}$ is the difference between the quasi-Fermi levels for electrons and holes.

\section{A.2 Spontaneous emission rate $R_{s p}(\hbar \omega)$}

Similarly, the spontaneous emission rate $R_{\mathrm{sp}}(\hbar \omega)$ per unit volume of the quantum transition involving the emission of a photon with energy $\hbar \omega$ and a phonon with energy $\hbar \Omega$ is written as follows ${ }^{36)}$

$$
R_{\mathrm{sp}}(\hbar \omega)=A_{\mathrm{cv}} \cdot \int_{E_{\mathrm{c}}} \int_{E_{\mathrm{v}}}\left(n_{\mathrm{q}}+1\right) \cdot D_{\mathrm{c}}\left(E_{\mathrm{c}}\right) D_{\mathrm{v}}\left(E_{\mathrm{v}}\right) f_{\mathrm{c}}\left(E_{\mathrm{c}}\right)\left[1-f_{\mathrm{v}}\left(E_{\mathrm{v}}\right)\right] \cdot \delta\left(\hbar \omega-E_{\mathrm{c}}+E_{\mathrm{v}}+\hbar \Omega\right) d E_{\mathrm{v}} d E_{\mathrm{c}}
$$

where $A_{\mathrm{cv}}$ is the spontaneous emission coefficient. If we again apply Boltzmann approximation to the distribution functions $f_{\mathrm{c}}$ and $\left(1-f_{\mathrm{v}}\right)$, the spontaneous emission rate $R_{\mathrm{sp}}(\hbar \omega)$ is reduced to the following simplified form

$$
R_{\mathrm{sp}}(\hbar \omega)=A_{\mathrm{cv}} \cdot N_{\mathrm{c}} N_{\mathrm{v}} \cdot\left(n_{\mathrm{q}}+1\right) \cdot \frac{\pi}{8}\left(\hbar \omega+\hbar \Omega-E_{\mathrm{g}}\right)^{2} \cdot \exp \left[-(\hbar \omega+\hbar \Omega-\Delta F) / k_{\mathrm{B}} T\right]
$$




\section{A.3 Absorption rate $R_{a b}(\hbar \omega)$}

Lastly, the absorption rate $R_{\mathrm{ab}}(\hbar \omega)$ per unit volume of the quantum transition involving the absorption of a photon with energy $\hbar \omega$ and a phonon with energy $\hbar \Omega$ takes the following form ${ }^{36)}$

$$
R_{\mathrm{ab}}(\hbar \omega)=B_{\mathrm{vc}} \cdot \rho_{\mathrm{ph}}(\hbar \omega) \cdot \int_{E_{\mathrm{c}}} \int_{E_{\mathrm{v}}} n_{\mathrm{q}} \cdot D_{\mathrm{c}}\left(E_{\mathrm{c}}\right) D_{\mathrm{v}}\left(E_{\mathrm{v}}\right)\left[1-f_{\mathrm{c}}\left(E_{\mathrm{c}}\right)\right] f_{\mathrm{v}}\left(E_{\mathrm{v}}\right) \cdot \delta\left(\hbar \omega-E_{\mathrm{c}}+E_{\mathrm{v}}+\hbar \Omega\right) d E_{\mathrm{v}} d E_{\mathrm{c}}
$$

where $B_{\mathrm{vc}}$ is the coefficient for absorption. The $n_{\mathrm{q}}$ term is associated with the probability of phonon absorption. ${ }^{36)}$ The delta function in the integral again restricts the energy $\left(E_{\mathrm{c}}-E_{\mathrm{v}}\right)$ to be equal to $(\hbar \omega+\hbar \Omega)$ due to the absorption of a photon and a phonon. Again, when the quasi-Fermi levels for electrons and holes locate in the bandgap far away from the conduction and valence band edges, the distribution functions $\left(1-f_{\mathrm{c}}\right)$ and $f_{\mathrm{v}}$ can be approximated by

$$
\begin{aligned}
1-f_{\mathrm{c}}\left(E_{\mathrm{c}}\right) & =\frac{1}{1+\exp \left[\left(F_{\mathrm{e}}-E_{\mathrm{c}}\right) / k_{\mathrm{B}} T\right]} \approx 1, \\
f_{\mathrm{v}}\left(E_{\mathrm{v}}\right) & =\frac{1}{1+\exp \left[\left(E_{\mathrm{v}}-F_{\mathrm{h}}\right) / k_{\mathrm{B}} T\right]} \approx 1 .
\end{aligned}
$$

Thus, eq. (A.8) is reduced to the following simple form:

$$
\begin{aligned}
R_{\mathrm{ab}}(\hbar \omega) & =B_{\mathrm{vc}} \cdot \rho_{\mathrm{ph}}(\hbar \omega) \cdot n_{\mathrm{q}} \cdot N_{\mathrm{c}} N_{\mathrm{v}} \int_{E_{\mathrm{c}}} \int_{E_{\mathrm{v}}}\left(E_{\mathrm{c}}-E_{\mathrm{g}}\right)^{1 / 2}\left(-E_{\mathrm{v}}\right)^{1 / 2} \cdot \delta\left(\hbar \omega-E_{\mathrm{c}}+E_{\mathrm{v}}+\hbar \Omega\right) d E_{\mathrm{v}} d E_{\mathrm{c}} \\
& =B_{\mathrm{vc}} \cdot N_{\mathrm{c}} N_{\mathrm{v}} \cdot \rho_{\mathrm{ph}}(\hbar \omega) \cdot n_{\mathrm{q}} \cdot \frac{\pi}{8}\left(\hbar \omega+\hbar \Omega-E_{\mathrm{g}}\right)^{2} .
\end{aligned}
$$

\section{A.4 Optical gain $g(\hbar \omega)$}

At thermodynamic equilibrium there is only one common Fermi level, i.e., $\Delta F=0$, and the Fermi level is located far away from conduction and valence band edges. As a result, the Boltzmann distribution represents valid approximation for the distribution functions $f_{\mathrm{c}}$ and $f_{\mathrm{v}}$. Now, the principle of detailed balance requires that the upward transition rate must equal the downward transition rate at thermodynamic equilibrium

$$
R_{\mathrm{ab}}(\hbar \omega)=R_{\mathrm{sp}}(\hbar \omega)+R_{\mathrm{st}}(\hbar \omega)
$$

Using eqs. (A.5), (A.7), and (A.10), the above equation yields

$$
B_{\mathrm{vc}} \rho_{\mathrm{ph}}(\hbar \omega) n_{\mathrm{q}}=\left[B_{\mathrm{cv}} \rho_{\mathrm{ph}}(\hbar \omega)+A_{\mathrm{cv}}\right] \cdot\left(n_{\mathrm{q}}+1\right) \cdot \exp \left(-\frac{\hbar \omega+\hbar \Omega}{k_{\mathrm{B}} T}\right)
$$

Since $n_{\mathrm{q}}$ is given by Bose-Einstein distribution, i.e., eq. (A.4), at thermodynamic equilibrium the common factors involving phonons such as $n_{\mathrm{q}}$ and $\hbar \Omega$ in eq. (A.12) cancel out and the following simple expression is obtained

$$
\rho_{\mathrm{ph}}(\hbar \omega)=\frac{A_{\mathrm{cv}}}{B_{\mathrm{vc}} \exp \left(\hbar \omega / k_{\mathrm{B}} T\right)-B_{\mathrm{cv}}}=D_{\mathrm{p}} n_{\mathrm{p}}=\frac{8 \pi n^{2} n_{\mathrm{g}}(\hbar \omega)^{2}}{h^{3} c^{3}} \frac{1}{\exp \left(\hbar \omega / k_{\mathrm{B}} T\right)-1}
$$

where the photon density per energy interval $\rho_{\mathrm{ph}}(\hbar \omega)$ is given by Plank's law, $D_{\mathrm{p}}=8 \pi n^{2} n_{\mathrm{g}}(\hbar \omega)^{2} / h^{3} c^{3}$ is the density of states of photon per energy interval, $n_{\mathrm{p}}=\left[\exp \left(\hbar \omega / k_{\mathrm{B}} T\right)-1\right]^{-1}$ is the photon occupation number which gives the average number of photons per state with the photon energy $\hbar \omega$ at thermodynamic equilibrium, $c$ is the velocity of light in free space, and $n$ and $n_{\mathrm{g}}$ are the refractive index and the group index of the materials, respectively. We see that

$$
\begin{aligned}
& B_{\mathrm{vc}}=B_{\mathrm{cv}}, \\
& A_{\mathrm{cv}}=D_{\mathrm{p}} B_{\mathrm{cv}} .
\end{aligned}
$$

Clearly, eqs. (A-14a) and (A·14b) are the same as the well-known results of Einstein's treatment for a simple two-level system. Thus the relationship between $A_{\mathrm{cv}}, B_{\mathrm{cv}}$, and $B_{\mathrm{vc}}$ in the phonon-assisted optical transitions are the same as that in a simple two-level system.

Now, rewriting the expressions for stimulated emission rate $R_{\mathrm{st}}(\hbar \omega)$ and absorption rate $R_{\mathrm{ab}}(\hbar \omega)$ in terms of spontaneous emission rate $R_{\mathrm{sp}}(\hbar \omega)$ yields

$$
\begin{aligned}
R_{\mathrm{st}}(\hbar \omega)= & \frac{B_{\mathrm{cv}}}{A_{\mathrm{cv}}} \cdot \rho_{\mathrm{ph}}(\hbar \omega) \cdot R_{\mathrm{sp}}(\hbar \omega) \\
R_{\mathrm{ab}}(\hbar \omega)= & B_{\mathrm{vc}} \cdot \rho_{\mathrm{ph}}(\hbar \omega) \cdot n_{\mathrm{q}} \\
& \times \int_{E_{\mathrm{c}}} \int_{E_{\mathrm{v}}} D_{\mathrm{c}}\left(E_{\mathrm{c}}\right) D_{\mathrm{v}}\left(E_{\mathrm{v}}\right)\left[1-f_{\mathrm{c}}\left(E_{\mathrm{c}}\right)\right] f_{\mathrm{v}}\left(E_{\mathrm{v}}\right) \cdot \delta\left(\hbar \omega-E_{\mathrm{c}}+E_{\mathrm{v}}+\hbar \Omega\right) d E_{\mathrm{v}} d E_{\mathrm{c}} \\
= & \left(B_{\mathrm{vc}} / A_{\mathrm{cv}}\right) \cdot \rho_{\mathrm{ph}}(\hbar \omega) \cdot n_{\mathrm{q}} /\left(n_{\mathrm{q}}+1\right) \cdot A_{\mathrm{cv}} \cdot\left(n_{\mathrm{q}}+1\right) \\
& \times \int_{E_{\mathrm{c}}} \int_{E_{\mathrm{v}}} D_{\mathrm{c}}\left(E_{\mathrm{c}}\right) D_{\mathrm{v}}\left(E_{\mathrm{v}}\right) f_{\mathrm{c}}\left(E_{\mathrm{c}}\right)\left[1-f_{\mathrm{v}}\left(E_{\mathrm{v}}\right)\right] \cdot \delta\left(\hbar \omega-E_{\mathrm{c}}+E_{\mathrm{v}}+\hbar \Omega\right) \cdot \frac{\left[1-f_{\mathrm{c}}\left(E_{\mathrm{c}}\right)\right] f_{\mathrm{v}}\left(E_{\mathrm{v}}\right)}{f_{\mathrm{c}}\left(E_{\mathrm{c}}\right)\left[1-f_{\mathrm{v}}\left(E_{\mathrm{v}}\right)\right]} d E_{\mathrm{v}} d E_{\mathrm{c}}
\end{aligned}
$$




$$
\begin{aligned}
= & \left(B_{\mathrm{vc}} / A_{\mathrm{cv}}\right) \cdot \rho_{\mathrm{ph}}(\hbar \omega) \cdot n_{\mathrm{q}} /\left(n_{\mathrm{q}}+1\right) \cdot A_{\mathrm{cv}} \cdot\left(n_{\mathrm{q}}+1\right) \\
& \times \int_{E_{\mathrm{c}}} \int_{E_{\mathrm{v}}} D_{\mathrm{c}}\left(E_{\mathrm{c}}\right) D_{\mathrm{v}}\left(E_{\mathrm{v}}\right) f_{\mathrm{c}}\left(E_{\mathrm{c}}\right)\left[1-f_{\mathrm{v}}\left(E_{\mathrm{v}}\right)\right] \cdot \delta\left(\hbar \omega-E_{\mathrm{c}}+E_{\mathrm{v}}+\hbar \Omega\right) \cdot \exp \left[\left(E_{\mathrm{c}}-E_{\mathrm{v}}-\Delta F\right) / k_{\mathrm{B}} T\right] d E_{\mathrm{v}} d E_{\mathrm{c}} \\
= & \left(B_{\mathrm{vc}} / A_{\mathrm{cv}}\right) \cdot \rho_{\mathrm{ph}}(\hbar \omega) \cdot n_{\mathrm{q}} /\left(n_{\mathrm{q}}+1\right) \cdot \exp \left[(\hbar \omega+\hbar \Omega-\Delta F) / k_{\mathrm{B}} T\right] \cdot A_{\mathrm{cv}} \cdot\left(n_{\mathrm{q}}+1\right) \\
& \times \int_{E_{\mathrm{c}}} \int_{E_{\mathrm{v}}} D_{\mathrm{c}}\left(E_{\mathrm{c}}\right) D_{\mathrm{v}}\left(E_{\mathrm{v}}\right) f_{\mathrm{c}}\left(E_{\mathrm{c}}\right)\left[1-f_{\mathrm{v}}\left(E_{\mathrm{v}}\right)\right] \cdot \delta\left(\hbar \omega-E_{\mathrm{c}}+E_{\mathrm{v}}+\hbar \Omega\right) d E_{\mathrm{v}} d E_{\mathrm{c}} \\
= & \frac{B_{\mathrm{vc}}}{A_{\mathrm{cv}}} \cdot \rho_{\mathrm{ph}}(\hbar \omega) \cdot \frac{n_{\mathrm{q}}}{n_{\mathrm{q}}+1} \cdot \exp \left(\frac{\hbar \omega+\hbar \Omega-\Delta F}{k_{\mathrm{B}} T}\right) \cdot R_{\mathrm{sp}}(\hbar \omega) .
\end{aligned}
$$

Although the relationships between $A_{\mathrm{cv}}, B_{\mathrm{cv}}$, and $B_{\mathrm{vc}}$ in eq. (A.14) are established under the condition of thermodynamic equilibrium, we assume that they remain valid even under the non-equilibrium conditions to derive optical gain. ${ }^{37,38)}$ Using eqs. (A·14)-(A·16), the optical gain $g(\hbar \omega)$ of the quantum transition involving the photon energy $\hbar \omega$ and the phonon energy $\hbar \Omega$ is obtained as follows

$$
\begin{aligned}
g(\hbar \omega) & \equiv \frac{R_{\mathrm{st}}(\hbar \omega)-R_{\mathrm{ab}}(\hbar \omega)}{\rho_{\mathrm{ph}}(\hbar \omega) \cdot\left(c / n_{\mathrm{g}}\right)} \\
& =\frac{h^{3} c^{2}}{8 \pi n^{2}(\hbar \omega)^{2}} R_{\mathrm{sp}}(\hbar \omega) \cdot\left[1-\frac{n_{\mathrm{q}}}{n_{\mathrm{q}}+1} \cdot \exp \left(\frac{\hbar \omega+\hbar \Omega-\Delta F}{k_{\mathrm{B}} T}\right)\right] .
\end{aligned}
$$

It should be noted that at thermodynamic equilibrium, $\Delta F=0$ and $n_{\mathrm{q}}$ is given by the Bose-Einstein statistics. ${ }^{36)}$ As a result, the optical gain becomes negative, i.e., absorption, and eq. (A.17) is reduced to the well-known van RoosbroeckShockley relation ${ }^{39}$ )

$$
R_{\mathrm{sp}}(\hbar \omega)=\alpha(\hbar \omega) \cdot \frac{8 \pi n^{2}(\hbar \omega)^{2}}{h^{3} c^{2}} \frac{1}{\exp \left(\hbar \omega / k_{\mathrm{B}} T\right)-1}
$$

where $\alpha(\hbar \omega)$ is the absorption coefficient. Clearly, eq. (A-18) provides a direct link between the absorption coefficient and the spontaneous emission rate under thermodynamic equilibrium situations.

After substituting eq. (A.7) into eq. (26a) and set $\Delta F=0$, the absorption coefficient $\alpha_{1}(\hbar \omega)$ associated with the onephonon assisted optical transition is obtained as follows:

$$
\begin{aligned}
\alpha_{1}(\hbar \omega) & =\frac{h^{3} c^{2}}{8 \pi n^{2}(\hbar \omega)^{2}} A_{\mathrm{cv} 1} \cdot N_{\mathrm{c}} N_{\mathrm{v}} \cdot\left(n_{\mathrm{q} 1}+1\right) \cdot \frac{\pi}{8}\left(\hbar \omega+\hbar \Omega_{1}-E_{\mathrm{g}}\right)^{2} \exp \left[-\left(\hbar \omega+\hbar \Omega_{1}\right) / k_{\mathrm{B}} T\right]\left[\exp \left(\hbar \omega / k_{\mathrm{B}} T\right)-1\right] \\
& =C_{1} \cdot \frac{1}{(\hbar \omega)^{2}} \cdot \frac{1}{1-\exp \left(-\hbar \Omega_{1} / k_{\mathrm{B}} T\right)} \cdot \frac{\pi}{8}\left(\hbar \omega+\hbar \Omega_{1}-E_{\mathrm{g}}\right)^{2} \exp \left[-\left(\hbar \omega+\hbar \Omega_{1}\right) / k_{\mathrm{B}} T\right]\left[\exp \left(\hbar \omega / k_{\mathrm{B}} T\right)-1\right]
\end{aligned}
$$

where the proportional constant $C_{1}=\left(h^{3} c^{2} / 8 \pi n^{2}\right) \cdot A_{\mathrm{cv} 1} \cdot N_{\mathrm{c}} N_{\mathrm{v}}$. According to eqs. (26a) and (A.6), the optical gain associated with the one-phonon assisted process can be rewritten in terms of the constant $C_{1}$ as follows

$$
\begin{aligned}
g_{1}(\hbar \omega)= & C_{1} \cdot \frac{1}{(\hbar \omega)^{2}} \cdot \frac{1}{1-\exp \left(-\hbar \Omega_{1} / k_{\mathrm{B}} T\right)} \cdot\left\{1-\exp \left[(\hbar \omega-\Delta F) / k_{\mathrm{B}} T\right]\right\} \\
& \times \int_{E_{\mathrm{c}}} \int_{E_{\mathrm{v}}}\left[E_{\mathrm{c}}-\left(E_{\mathrm{g}}-\Delta E_{\mathrm{g}}\right)\right]^{1 / 2}\left(-E_{\mathrm{v}}\right)^{1 / 2} f_{\mathrm{c}}\left(E_{\mathrm{c}}\right)\left[1-f_{\mathrm{v}}\left(E_{\mathrm{v}}\right)\right] \cdot \delta\left(\hbar \omega-E_{\mathrm{c}}+E_{\mathrm{v}}+\hbar \Omega_{1}\right) d E_{\mathrm{v}} d E_{\mathrm{c}}
\end{aligned}
$$

\section{Appendix B}

It is necessary to relate the rates of stimulated emission, spontaneous emission and absorption to the electron and hole concentrations. The spontaneous emission rate as defined in eq. (A.6) is related to the concentrations of electrons and holes as follows:

$$
\begin{aligned}
R_{\mathrm{sp}}(\hbar \omega) & =B(\hbar \omega) \cdot N P \\
& =A_{\mathrm{cv}} \cdot\left(n_{\mathrm{q}}+1\right) \cdot N_{\mathrm{c}} N_{\mathrm{v}} \int_{E_{\mathrm{c}}} \int_{E_{\mathrm{v}}}\left(E_{\mathrm{c}}-E_{\mathrm{g}}\right)^{1 / 2}\left(-E_{\mathrm{v}}\right)^{1 / 2} f_{\mathrm{c}}\left(E_{\mathrm{c}}\right)\left[1-f_{\mathrm{v}}\left(E_{\mathrm{v}}\right)\right] \cdot \delta\left(\hbar \omega-E_{\mathrm{c}}+E_{\mathrm{v}}+\hbar \Omega\right) d E_{\mathrm{v}} d E_{\mathrm{c}} \\
& =A_{\mathrm{cv}} \cdot\left(n_{\mathrm{q}}+1\right) \cdot N_{\mathrm{c}} N_{\mathrm{v}} \cdot I
\end{aligned}
$$

where $B(\hbar \omega)$ is the bimolecular radiative recombination coefficient, ${ }^{34)} N$ and $P$ are the electron and hole concentrations, and the integral in eq. (B.1) is denoted by $I$, respectively. Accordingly,

$$
B(\hbar \omega)=A_{\mathrm{cv}} \cdot\left(n_{\mathrm{q}}+1\right) \cdot N_{\mathrm{c}} N_{\mathrm{v}} \cdot \frac{I}{N P} .
$$

Since $B(\hbar \omega)$ is independent of $\Delta F,{ }^{40)}$ we can calculate the value of $I / N P$ in eq. $(\mathrm{B} \cdot 2)$ in the region where Boltzmann approximation is valid. Using eqs. (A.2), (A.3), and (A.5), we obtain 


$$
\frac{I}{N P}=\frac{\frac{\pi}{8}\left(\hbar \omega+\hbar \Omega-E_{\mathrm{g}}\right)^{2} \exp \left(-\frac{\hbar \omega+\hbar \Omega-\Delta F}{k_{\mathrm{B}} T}\right)}{N_{\mathrm{c}} N_{\mathrm{v}} \exp \left(-\frac{E_{\mathrm{g}}-\Delta F}{k_{\mathrm{B}} T}\right)}=\frac{1}{N_{\mathrm{c}} N_{\mathrm{v}}} \frac{\pi}{8}\left(\hbar \omega+\hbar \Omega-E_{\mathrm{g}}\right)^{2} \exp \left(-\frac{\hbar \omega+\hbar \Omega-E_{\mathrm{g}}}{k_{\mathrm{B}} T}\right) .
$$

Thus the bimolecular radiative recombination coefficient $B(\hbar \omega)$ and the spontaneous emission rate $R_{\mathrm{sp}}(\hbar \omega)$ are given by

$$
\begin{aligned}
B(\hbar \omega) & =\frac{\pi}{8} A_{\mathrm{cv}}\left(n_{\mathrm{q}}+1\right)\left(\hbar \omega+\hbar \Omega-E_{\mathrm{g}}\right)^{2} \exp \left(-\frac{\hbar \omega+\hbar \Omega-E_{\mathrm{g}}}{k_{\mathrm{B}} T}\right)=M \cdot\left(n_{\mathrm{q}}+1\right), \\
R_{\mathrm{sp}}(\hbar \omega) & =\frac{\pi}{8} A_{\mathrm{cv}}\left(n_{\mathrm{q}}+1\right)\left(\hbar \omega+\hbar \Omega-E_{\mathrm{g}}\right)^{2} \exp \left(-\frac{\hbar \omega+\hbar \Omega-E_{\mathrm{g}}}{k_{\mathrm{B}} T}\right) N P=M \cdot\left(n_{\mathrm{q}}+1\right) \cdot N P
\end{aligned}
$$

where the proportional constant $M=\pi / 8 \cdot A_{\mathrm{cv}}\left(\hbar \omega+\hbar \Omega-E_{\mathrm{g}}\right)^{2} \exp \left[-\left(\hbar \omega+\hbar \Omega-E_{\mathrm{g}}\right) / k_{\mathrm{B}} T\right]$. Similarly, for the stimulated emission rate eq. (A.15) and the absorption rate eq. (A.16),

$$
\begin{aligned}
R_{\mathrm{st}}(\hbar \omega) & =\frac{B_{\mathrm{cv}}}{A_{\mathrm{cv}}} \cdot \rho_{\mathrm{ph}}(\hbar \omega) \cdot R_{\mathrm{sp}}(\hbar \omega) \\
& =\frac{1}{D_{\mathrm{p}}} \cdot D_{\mathrm{p}} n_{\mathrm{p}} \cdot M\left(n_{\mathrm{q}}+1\right) N P \\
& =M \cdot n_{\mathrm{p}}\left(n_{\mathrm{q}}+1\right) \cdot N P, \\
R_{\mathrm{ab}}(\hbar \omega) & =\frac{B_{\mathrm{vc}}}{A_{\mathrm{cv}}} \cdot \rho_{\mathrm{ph}}(\hbar \omega) \cdot \frac{n_{\mathrm{q}}}{n_{\mathrm{q}}+1} \cdot \exp \left(\frac{\hbar \omega+\hbar \Omega-\Delta F}{k_{\mathrm{B}} T}\right) \cdot R_{\mathrm{sp}}(\hbar \omega) \\
& =\frac{1}{D_{\mathrm{p}}} \cdot D_{\mathrm{p}} n_{\mathrm{p}} \cdot \frac{n_{\mathrm{q}}}{n_{\mathrm{q}}+1} \cdot \exp \left(\frac{\hbar \omega+\hbar \Omega-\Delta F}{k_{\mathrm{B}} T}\right) \cdot M\left(n_{\mathrm{q}}+1\right) N P \\
& =M \cdot n_{\mathrm{p}} n_{\mathrm{q}} \cdot \exp \left(\frac{\hbar \omega+\hbar \Omega-\Delta F}{k_{\mathrm{B}} T}\right) \cdot N P .
\end{aligned}
$$

Note that $D_{\mathrm{p}}$ and $n_{\mathrm{p}}$ have been defined in connection with eq. (A.13).

\section{Appendix C}

By using eqs. (A-20), (26a), (28), and (29), the ratio of the optical gain coefficient to the absorption coefficient for onephonon assisted optical transition is given by

$$
\begin{aligned}
\frac{g_{1}(\hbar \omega)}{\alpha_{1}(\hbar \omega)}= & \frac{R_{\mathrm{sp} 1}(\hbar \omega, \Delta F \neq 0)}{R_{\mathrm{sp} 1}(\hbar \omega, \Delta F=0)} \cdot \frac{\left\{1-\exp \left[(\hbar \omega-\Delta F) / k_{\mathrm{B}} T\right]\right\}}{\left[\exp \left(\hbar \omega / k_{\mathrm{B}} T\right)-1\right]} \\
= & \frac{\int_{E_{\mathrm{c}}} \int_{E_{\mathrm{v}}}\left[E_{\mathrm{c}}-\left(E_{\mathrm{g}}-\Delta E_{\mathrm{g}}\right)\right]^{1 / 2}\left(-E_{\mathrm{v}}\right)^{1 / 2} f_{\mathrm{c}}\left(E_{\mathrm{c}}\right)\left[1-f_{\mathrm{v}}\left(E_{\mathrm{v}}\right)\right] \cdot \delta\left(\hbar \omega-E_{\mathrm{c}}+E_{\mathrm{v}}+\hbar \Omega_{1}\right) d E_{\mathrm{v}} d E_{\mathrm{c}}}{\pi / 8 \cdot\left(\hbar \omega+\hbar \Omega_{1}-E_{\mathrm{g}}\right)^{2} \exp \left[-\left(\hbar \omega+\hbar \Omega_{1}\right) / k_{\mathrm{B}} T\right]} \\
& \times \frac{\left\{1-\exp \left[(\hbar \omega-\Delta F) / k_{\mathrm{B}} T\right]\right\}}{\left[\exp \left(\hbar \omega / k_{\mathrm{B}} T\right)-1\right]} .
\end{aligned}
$$

Under the condition of high carrier injection, i.e., $\Delta F \gg \hbar \omega$, the distribution functions $f_{\mathrm{c}}\left(E_{\mathrm{c}}\right) \approx 1$ and $\left[1-f_{\mathrm{v}}\left(E_{\mathrm{v}}\right)\right] \approx 1$, and so eq. $(\mathrm{C} \cdot 1)$ reduces to the following simple form:

$$
\begin{aligned}
\frac{g_{1}(\hbar \omega)}{\alpha_{1}(\hbar \omega)} & \approx \frac{\left(\hbar \omega+\hbar \Omega_{1}-E_{\mathrm{g}}+\Delta E_{\mathrm{g}}\right)^{2}}{\left(\hbar \omega+\hbar \Omega_{1}-E_{\mathrm{g}}\right)^{2}} \exp \left[\hbar \Omega_{1} / k_{\mathrm{B}} T\right] \\
& =\left(1+\frac{\Delta E_{\mathrm{g}}}{\hbar \omega+\hbar \Omega_{1}-E_{\mathrm{g}}}\right)^{2} \exp \left[\hbar \Omega_{1} / k_{\mathrm{B}} T\right] .
\end{aligned}
$$

The first factor in eq. (C.2) represents the effect of bandgap narrowing, while the second factor results from the involvement of phonons in optical transitions. ${ }^{22}$ Equation $(\mathrm{C} \cdot 2)$ clearly shows that both bandgap narrowing and phonon participation contribute to the large disparity between the maximum optical gain and the absorption coefficient in bulk crystalline silicon. 